Part of Journal of Research of the National Bureau of Standards, Volume 24, May 1940

\title{
EFFECT OF PAINT ON THE SOUND ABSORPTION OF ACOUSTIC MATERIALS
}

\author{
By V. L. Chrisler
}

\section{ABSTRACT}

A brief discussion is given of the properties of a material that cause it to absorb sound. Photographs are shown and the results of sound-absorption measurements are given for a number of different types of material before and after painting. A brief discussion is given of the kind of paint which should be used and how it should be applied to minimize the effect of the paint on the acoustical properties.

\section{CONTENTS}

I. Introduction

II. How sound is absorbed

(1) 548

III. Discussion of experimental results____ 549

IV. Summary _....... 552

\section{INTRODUCTION}

One of the serious maintenance problems which arises where acoustic treatment has been used is that of cleaning and redecorating. This is particularly true where indirect lighting is used, as a dirty ceiling decreases the illumination by a considerable amount. A search through the literature reveals very little information on this subject. Books on architectural acoustics and acoustic treatment barely mention the painting of acoustic material. The only paper dealing with the subject with which the author is familiar is one by F. C. Atwood. ${ }^{1}$ This article was published several years ago, and there have been many changes in acoustic materials since that time.

During the past few years a number of acoustic materials have been painted at the National Bureau of Standards and have been tested for sound absorption both before and after painting. As a result of this work, it has been found that when the materials are clean, that is, when the pores are not clogged with dirt, a number of coats of paint can be applied on many materials without seriously decreasing their sound absorption. If similar results are to be obtained on material which has been installed for several years, the dust should be cleaned thoroughly from the surface before the paint is applied.

The object of this paper is to indicate to what extent paint can be applied to different types of materials and, by a series of photographs, to show the appearance of the different surfaces before and after painting.

1 National Paint, Varnish, and Lacquer Association, Washington, D. C., The Valuation of Paints for Acoustical Treatments, Circular 445 (Nov. 1933). 


\section{HOW SOUND IS ABSORBED}

Before going into the details concerning the application of the paint, it is desirable to consider the different methods by which sound may be absorbed and what effect the application of paint can have on these different methods of absorption.

When one thinks of a sound-absorbent material he generally thinks of some material that is porous and in which the sound is absorbed because of the porosity of the material. A large number of the materials which are now sold as acoustic materials do depend to a considerable extent for their sound absorption on the porosity of the materials. A few of these materials have very large pores which are so interconnected that the sound absorption can be increased to a limited extent by partly closing the pore openings at the surfaces. Other materials have small openings which are not so well interconnected, with the result that the sound absorption is decreased as soon as any of the pore openings are closed. Owing to these differences, the effect of a coat of paint in changing the absorption due to porosity varies considerably for different types of material. For instance, a material with large pores may have the absorption increased by the first few coats of paint, but finally a stage will be reached where the absorption begins to decrease. The point where this loss in absorption begins depends upon the frequency of the sound, always showing up first at the higher frequencies. Materials with small pores which are not intercommunicating may have a considerable number of pores closed with the first coat of paint. In this case one would expect to find a decrease in the sound absorption of the material on painting.

Sound may also be absorbed by setting the material into damped vibration as a diaphragm, the more the damping the greater being the absorption. Likewise, a soft material may absorb sound by inelastic yielding. When the sound is absorbed by one of these methods, the effect of paint is quite different from that when it is applied to a material where the absorption is entirely due to porosity.

Where there is a damped vibration of a diaphragm, the maximum absorption occurs at a frequency equal to the natural frequency of the diaphragm and the absorption decreases at frequencies both above and below the natural frequency. When paint is applied, there is a slight increase in weight and in many cases an increase in stiffness. As a result of these changes, the frequency at which maximum absorption occurs will be changed; but in some cases the average absorption may remain about the same.

When the absorption occurs in a soft material because of an inelastic yield, this can be taken as a special case of a damped vibration of a diaphragm - that is, the natural frequency has approached zero. Today it is seldom that a material of this type is found, but a few years ago a very common treatment was hair felt on which was glued a painted membrane. After a few coats of paint were applied, the pores were completely sealed. Under these conditions most of the absorption was due to an inelastic yield of the felt. In this case additional coats of paint increased the weight and stiffness of the surface membrane and, as a result, decreased the absorption of the material. 


\section{DISCUSSION OF EXPERIMENTAL RESULTS}

Since the various methods by which sound is absorbed have been discussed, the next step is to determine how the different types of material are affected by the application of paint. All the samples described in this paper were painted with an interior flat white paint. In the past, other types of paints have been used, but the samples had not been preserved. A photographic study of those samples is therefore impossible. In examining the numerical results, it appears that approximately the same results are obtained whether a cold-water casein paint or an interior flat paint is used. It is apparent, however, that the manner in which the paint is applied is of considerable importance, except for materials having a perforated surface with the perforations so large that the paint will not film across the openings. For other materials to which the paint was applied with a spray gun, the results indicate that the paint should be applied as a fine spray and the quantity applied should not be sufficient to film across the fibers or grains in the acoustic material so as to form a solid film. A similar situation exists when the paint is applied with a brush. The paint should not be excessively thick and it should be applied in such a manner as to film across the pores as little as possible. Most of the results given in this paper are for samples to which the paint was applied with a brush, as this represents the worst condition. A much better painting job, as far as appearance is concerned, can be done on most acoustic materials with a spray gun, and as a rule the sound absorption is decreased by a smaller amount when the paint is applied with a spray gun than when applied with a brush.

The illustrations at the end of this paper show the extent to which the paint films across different types of surfaces. Table 1 gives the sound absorption coefficients of the materials shown in the photographs before and after painting, and table 2 gives the amount of paint used. The paint used in each case was a flat white interior oil paint and is one of the well-known brands of interior paint. When the paint was applied with a brush, it was thinned slightly by adding 10 percent of turpentine by volume, with the exception of sample 7 . In this case 25 percent of turpentine by volume was added. When applied with a spray gun, the paint was thinned by adding 50 percent of turpentine by volume. The quantities of paint given in table 2 are the quantities of mixed paint.

To determine the hiding power of the paint, it has been our practice to obtain a reasonably white surface, then to paint black stripes across the face of the acoustic material and determine the number of coats of white paint required to hide the black stripes. The results of this test are given in table 2.

Samples 1 and 2 are representative of materials made from a wood fiber similar to fine excelsior. They comply with the requirements in Federal Specification SS-A-118 for Type VII. As the surface of this material was not white, it was necessary to apply one coat of white paint before the black stripes were applied. The four additional coats of white paint indicated in table 2 failed to hide the black completely. To obtain complete hiding, it was necessary to apply one more coat of paint. If this material had been spray painted, it would have been easier to hide the black, the reason being that the black paint was applied with a $1 / 2$-in. brush, which covered the sides as well 
as the top of the fibers. When a larger brush was used for the subsequent white coats, the tendency was to paint only the top of the fibers. If the white paint had been applied with a spray gun, it would have been possible to cover the sides of the fiber and a much better result could have been obtained with less paint.

Figure 1 shows the general appearance of this type of material. Figure 2 shows the fibrous appearance of the surface magnified about $1 \%$ times. Figure 3 shows the appearance of the surface after five coats of paint have been applied with a brush.

The values of the sound-absorption coefficients for these samples given in table 1 are rather surprising. As indicated by figure 3 and shown by an examination of the material, a large number of the surface pores have been filled. As a result of this surface film, sample $1 a$ absorbs considerably less sound at the higher frequencies than the unpainted sample. This same effect is also noticeable on sample $2 a$, but to a smaller degree. The most surprising result is the decided increase of absorption at 512 cycles per second. It would appear that this type of material has a structure in which the space between the fibers are so open and intercommunicating that a maximum absorption can be obtained at this frequency by partially closing the surface pores. Such a statement is not true for most of the other types of materials.

When acoustic materials are used in offices or similar places for noise reduction, it has been noticed that the sensation of quietness depends to a considerable extent upon the acoustic material being efficient at the higher frequencies. This being the case, the actual value of an acoustic treatment which might be represented by sample $1 a$ has decreased more than would be indicated by the change of the noise coefficient from 0.60 to 0.55 .

Sample 3 is one of the incombustible materials of Type VI as described by Federal Specification SS-A-118. In this case the black stripes were again applied after the first coat of white paint. The four additional coats hid the black. Figure 4 shows the general appearance of this type of acoustic material. Figure 5 shows the surface appearance of this material magnified about $1 \% 3$ times. Figure 6 shows how the surface appears after five coats of paint have been applied with a brush.

It will be noticed that the fine surface pores have been closed and there has been some loss of absorption at the higher frequencies. The larger openings, which give the fissured effect, are still open, and it would require a considerable number of additional coats of paint to close these openings. It is also of interest to note that the internal structure of this material is different from samples 1 and 2 , and that there is not the large increase in absorption at 512 cycles.

Sample 4 , as shown in figure 7 , is representative of the material described as Type V in Federal Specification SS-A-118. Figure 8 shows the surface appearance of this material magnified about $1 \frac{2}{3}$ times and figure 9 the appearance after brush painting with four coats. The surface finish as received from the manufacturer was white, hence the black stripes were applied before the white coats of paint were applied.

Sample 5, as shown in figure 10 , is a material which is classified as Type VIII in Federal Specification SS-A-118. This sample had what has been termed a "brushed" surface and is not typical of the materials 
grouped under Type VIII. Many of the materials have a sanded surface, and when paint is applied it films over much more easily than on the sample shown as 5. Figure 11 shows the surface of this material magnified three times. This material is made of wood fibers, as were samples 1 and 2 , but of a much finer fiber; and when the fibers are felted together, the openings between the fibers are much smaller than for samples 1 and 2. Owing to the finer pores between the fibers, this material does not show an increase in absorption at 512 cycles when paint is applied. In fact, when it was brush painted with five coats, there was a decrease in absorption at this frequency; but at 128 cycles the absorption has been increased by the painting. It is also to be noticed that the brush painting increased the absorption at this frequency more than the spray painting.

Figures 12 and 13 have the same magnification as figure 11 and show the appearance of the surface of this type of material after it has been spray painted with seven coats and brush painted with five coats, respectively.

It can be seen that the spray painting has left the surface of this material quite open and that there is only a slight decrease in absorption at the higher frequencies. The brush painting has closed up a large portion of the surface, and on the average there has been a considerable decrease in sound absorption. When the material which was photographed for these prints is examined it is noted that the spray painting gives a much better appearing material than does brush painting. In fact, this is true of all of the materials discussed in this paper except samples 3 and 6 .

Sample 6 , as shown in figure 14 , is a material which has been classified as Type VI in Federal Specification SS-A-118. Figure 15 shows how this surface appears when it has been magnified $1 \frac{2}{3}$ times. This material was painted by the manufacturer. Figure 16 shows the appearance of the surface after it has received four additional coats of paint. Judging from the previous samples, it would not be expected that the absorption of this material would be appreciably decreased by the added paint; but referring to table 1, it is found that there is an appreciable loss of absorption particularly at the higher frequencies. This is probably due to the fact that pores between the relatively large particles are not as intercommunicating as those in the previous samples.

Samples 7 and 8 are of an acoustic material which is applied with an air gun, and the surface appearance after painting is shown in figures 17 and 18. The magnification in this case is two-thirds. This material is of such a nature that it can be applied to give a number of different surface textures. Figure 17 shows what the manufacturer termed a "travertine" finish and figure 18 a smooth finish. Both these samples were applied directly on metal lath. For the unpainted sample the absorption must take place by all three methods discussed in the first part of this paper, as the material is porous, soft, so that it yields easily to the sound pressure, and relatively thin and light, so that it vibrates easily as a diaphragm.

Sample $8 a$, which had been brush painted with four coats in addition to the paint applied by the manufacturer, had a paint film which was almost continuous, there being only an occasional opening about the size of a pinhead. This means that nearly all absorption by porosity was destroyed. Also, the paint film was so stiff that there was little 
yielding or vibration as a diaphragm at the higher frequencies, with the result that the absorption coefficients for these frequencies were quite small. At the lower frequencies this is not true, and the absorption has not been materially affected by the paint.

The principal difference between $7 a$ and $8 a$ is that the paint film is broken at many points on $7 a$. The surface of this sample was rough and had numerous depressions, which prevented the painter from obtaining a continuous paint film when the paint was applied with a $3 \frac{1}{2}$-in. brush in the usual manner. As a result of these openings, a considerable amount of the sound energy was absorbed because of the porosity of the material, and there was not as much loss in absorption at the higher frequencies as there was for sample $8 a$.

Sample 9 is representative of Type II materials as described by Federal Specification SS-A-118. The surface texture is also similar to that of most of the acoustic plasters. The absorption of this type of material is largely due to the porosity of the material. Figure 19 shows the appearance of the surface after it has been magnified $1 \%$ times. It will be noted that there are numerous small openings between the surface particles. Figure 20 shows the appearance of the surface after it has been brush painted with five coats. The small openings which could be seen before painting are now almost completely covered with a paint film. As shown in table 1, the absorption of this type of material is lost to a large extent as soon as sufficient paint is applied to form such a film.

It should be possible to paint this material more successfully with a spray gun, but considerable care should be taken not to apply too much paint in any one coat. After a few coats, however, such a material will film across with a resulting loss in absorption.

\section{SUMMARY}

The results given in this paper are not as complete as might be desired, but the materials on which results are given are quite representative of most of the acoustic materials which are on the market at the present time. Some of these materials can be painted with only one or two coats before there is a noticeable decrease in the sound absorption of the material, whereas other materials can be painted many times before the acoustic properties of the materials have been decreased. In practically all cases, however, considerable care must be taken as to how the paint is applied.

A comparison of the results given in this paper with the results of various paint tests which have been published in the Bureau Letter Circulars on sound absorption coefficients issued during the past 10 years indicates that about the same results are obtained, whether the paint is a cold-water paint or an interior flat paint. In other words, if the paint is thinned to some extent before being applied and each coat is applied thin enough so as not to form a film across the surface, the principal factor which affects the decrease in absorption is the amount of pigment that is deposited on the surface. When a thin coat of paint is applied, most of this pigment is deposited on the top and sides of the surface particles or small projections on the surface of the acoustic materials. Additional coats continue to build up this amount of pigment. When the pore openings are very small, one or two coats may be sufficient to build this pigment deposit across the 


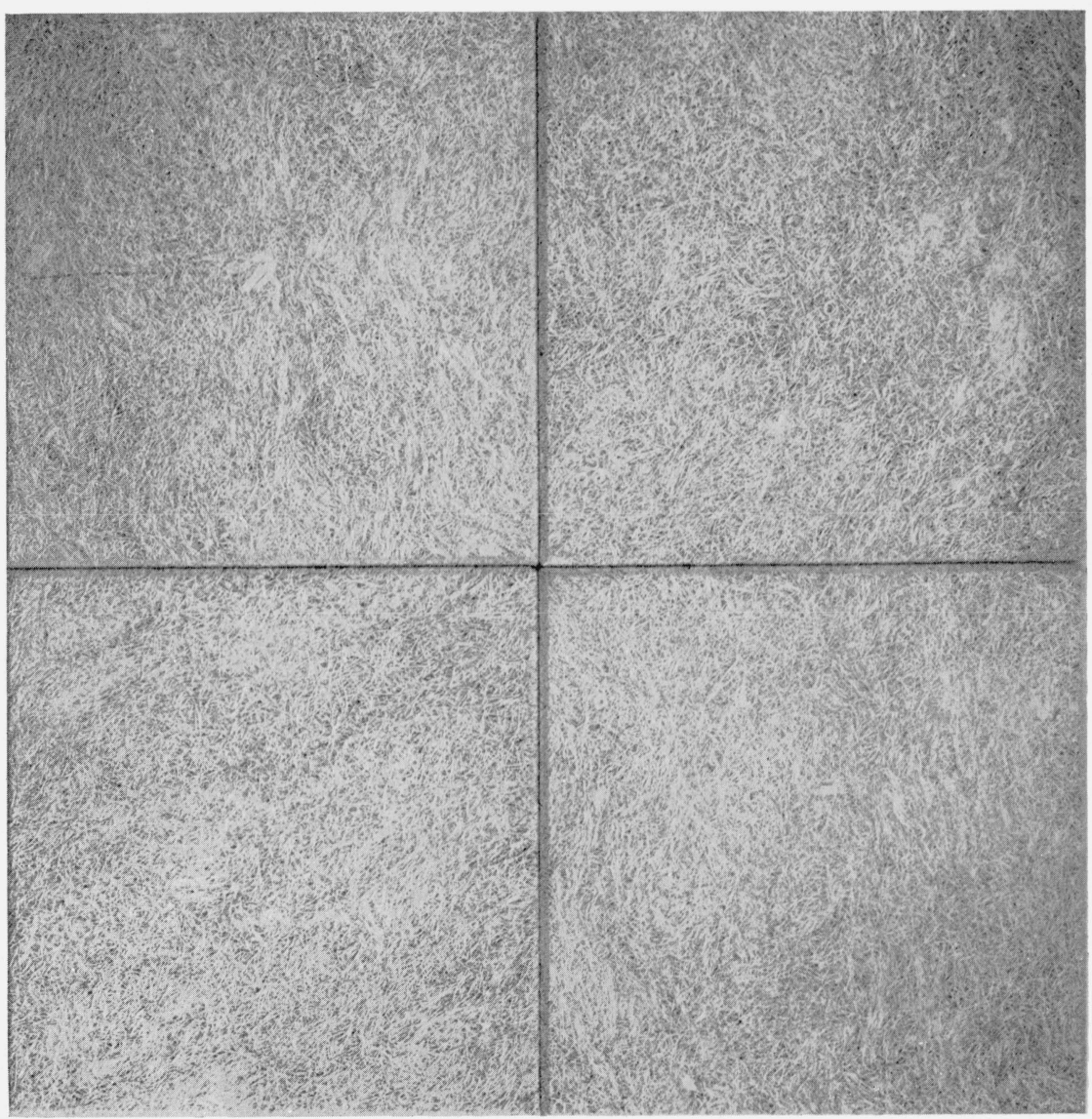

FiguRE 1.-General appearance of samples 1 and 2. 


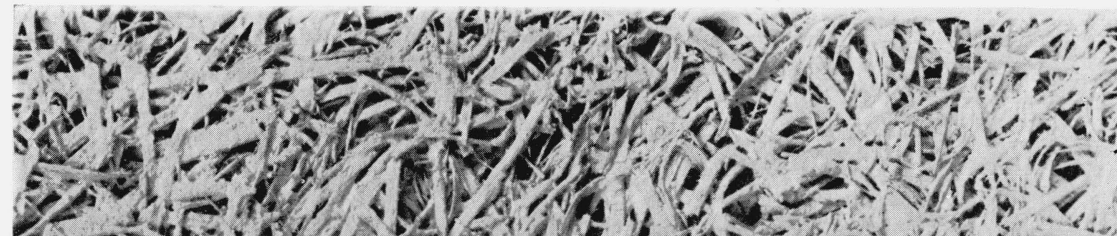

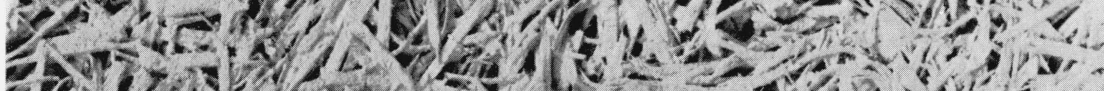

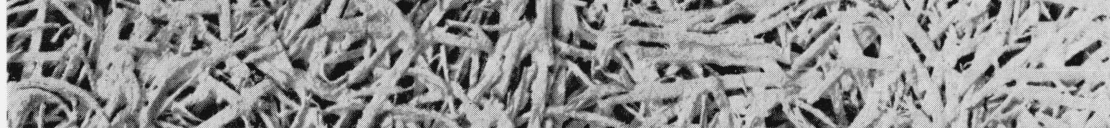

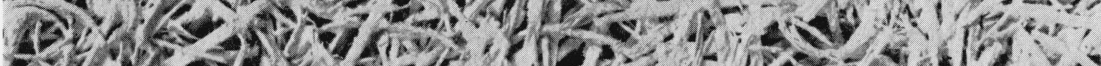

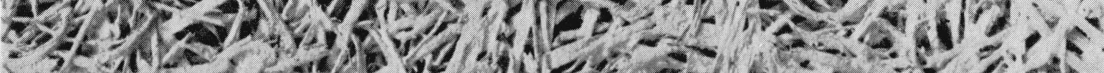

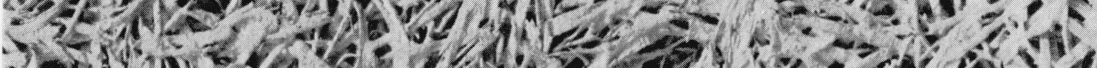
- I.

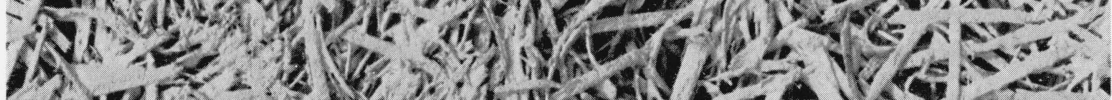
(1)

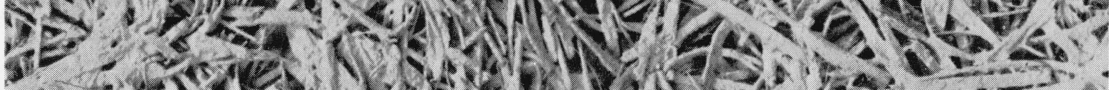

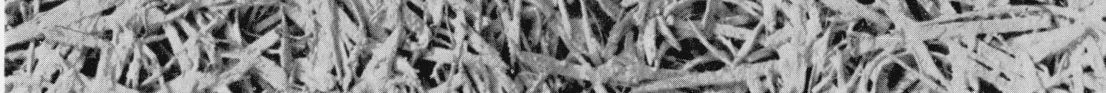

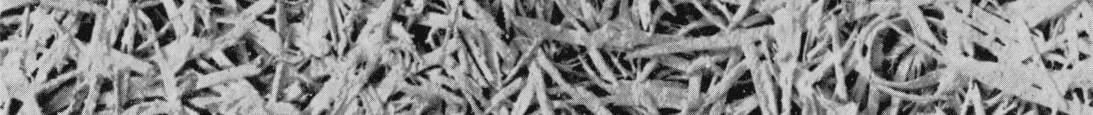

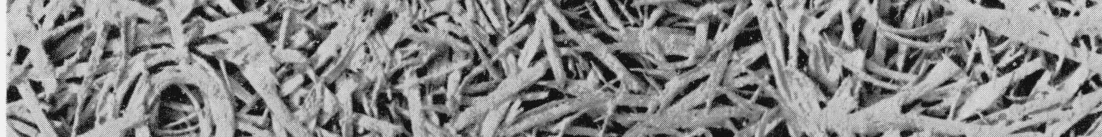

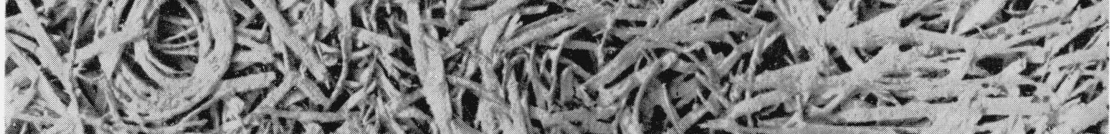

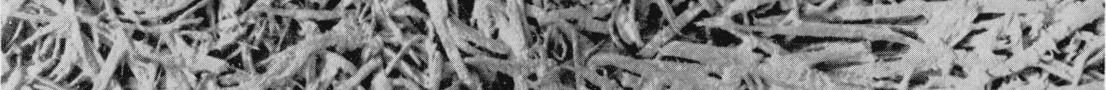

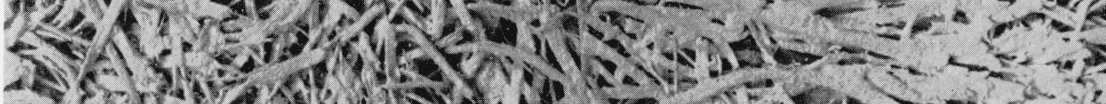

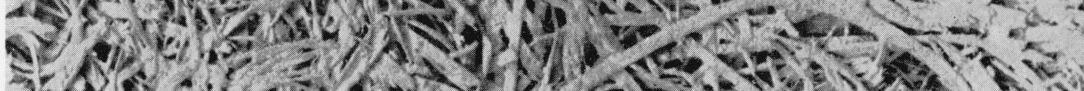

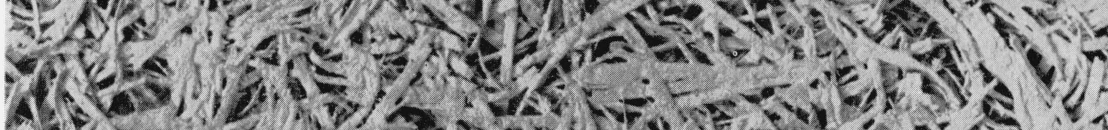

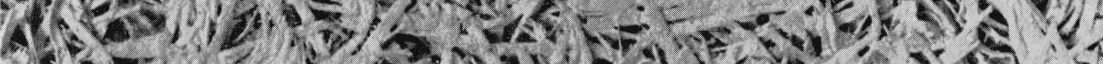
35)

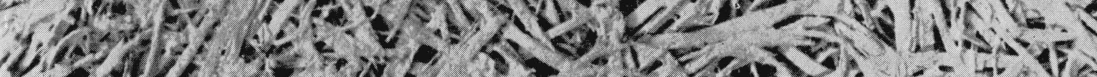

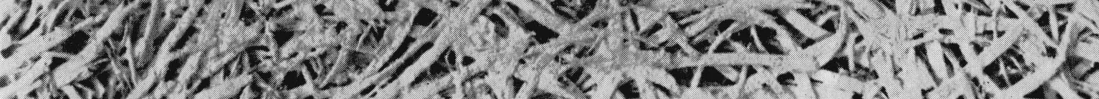

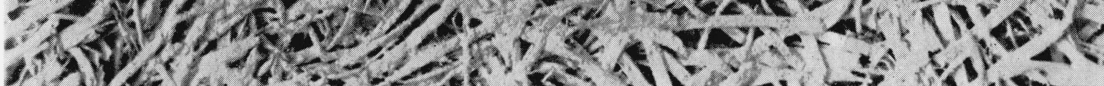

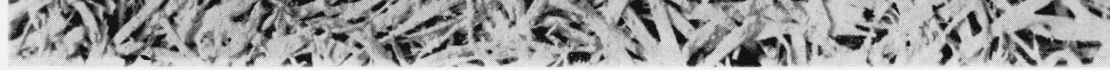

FigURE 2.-Surface appearance of samples 1 and 2 when unpainted and magnified $12 \frac{1}{3}$ times. 


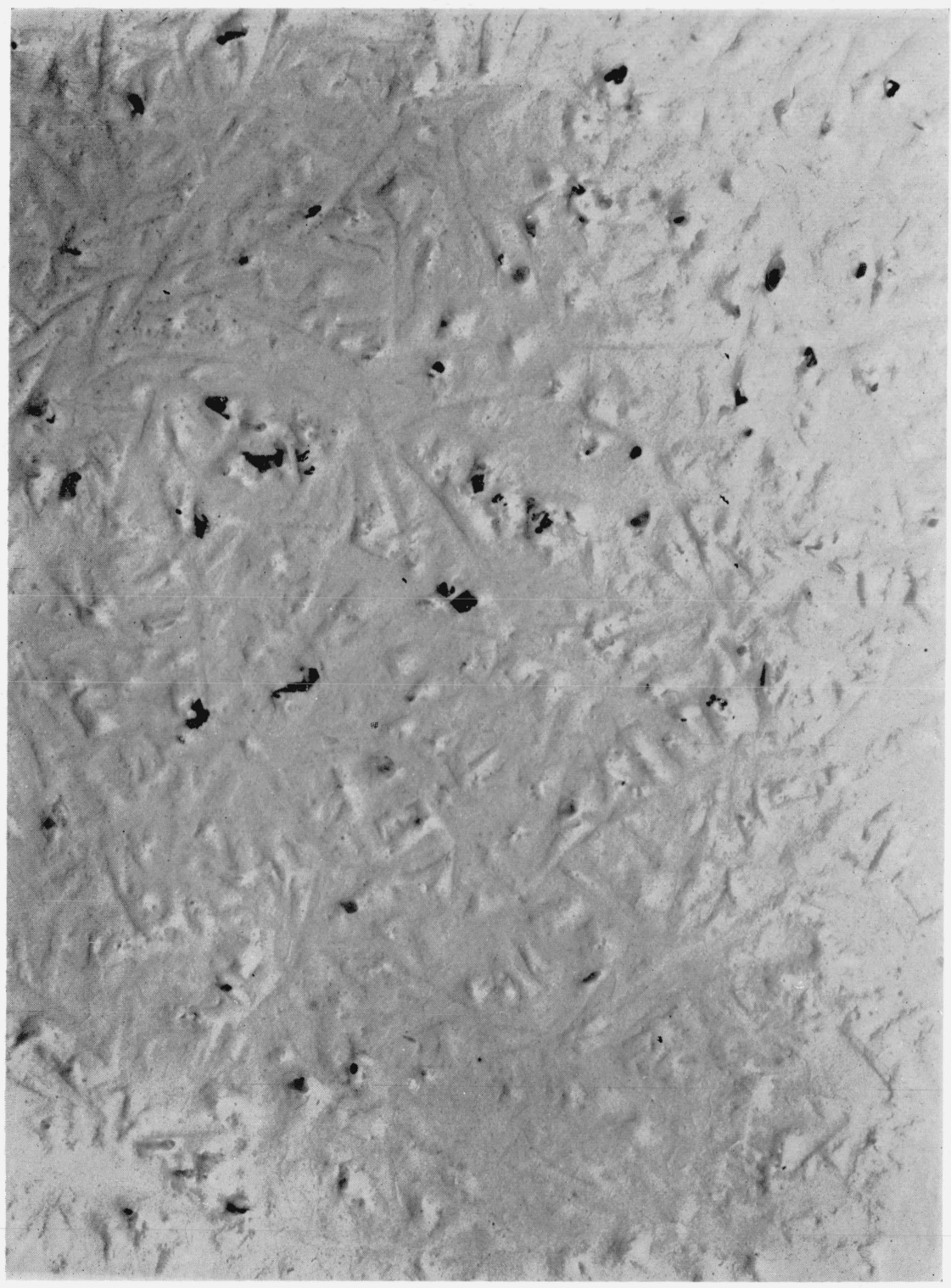

FIGURE 3.-Surface appearance of samples $1 a$ and $2 a$ when brush painted five coats and magnified $1 \frac{2}{3}$ times. 


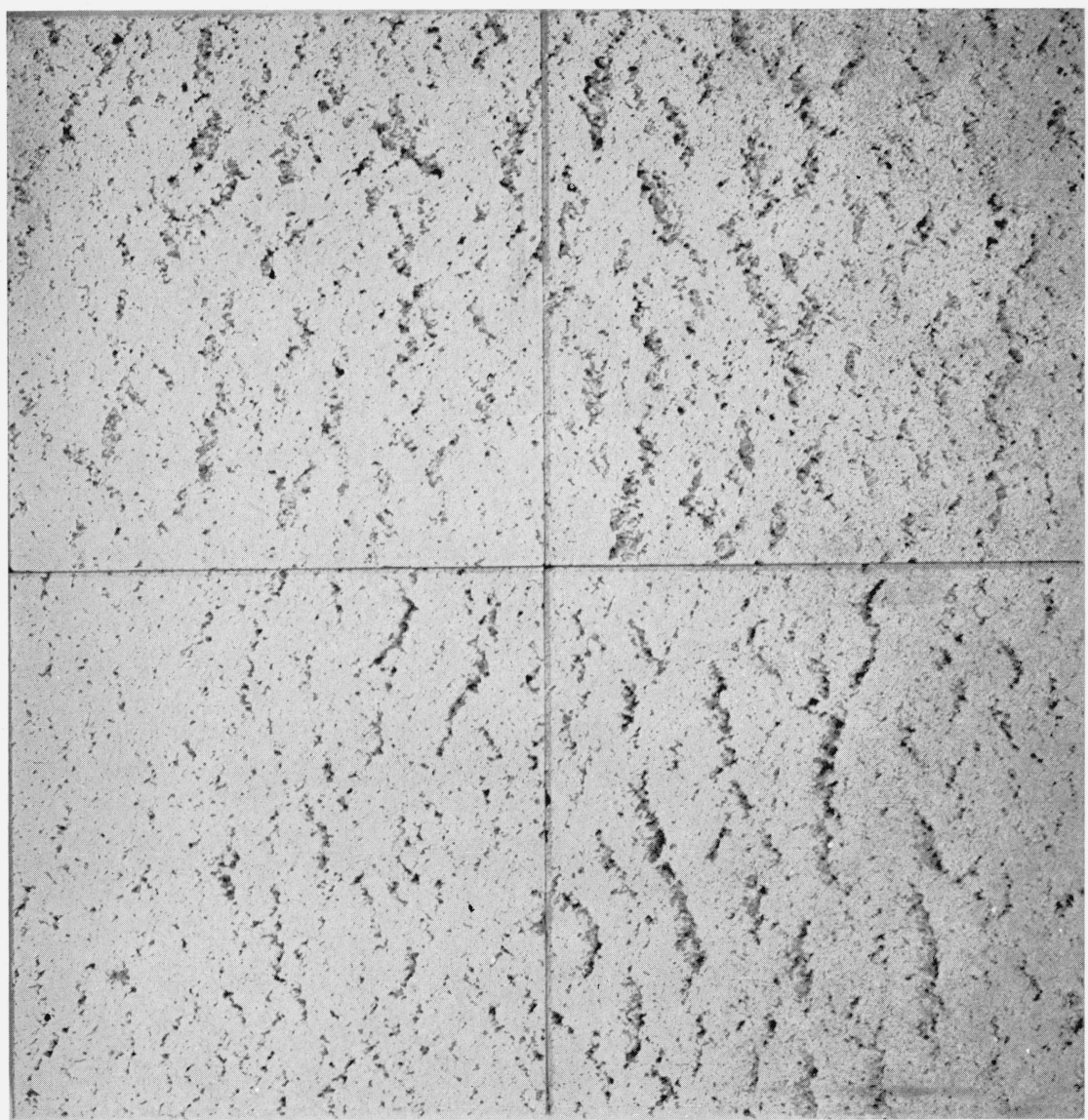

Figure 4.-General appearance of sample 3. 


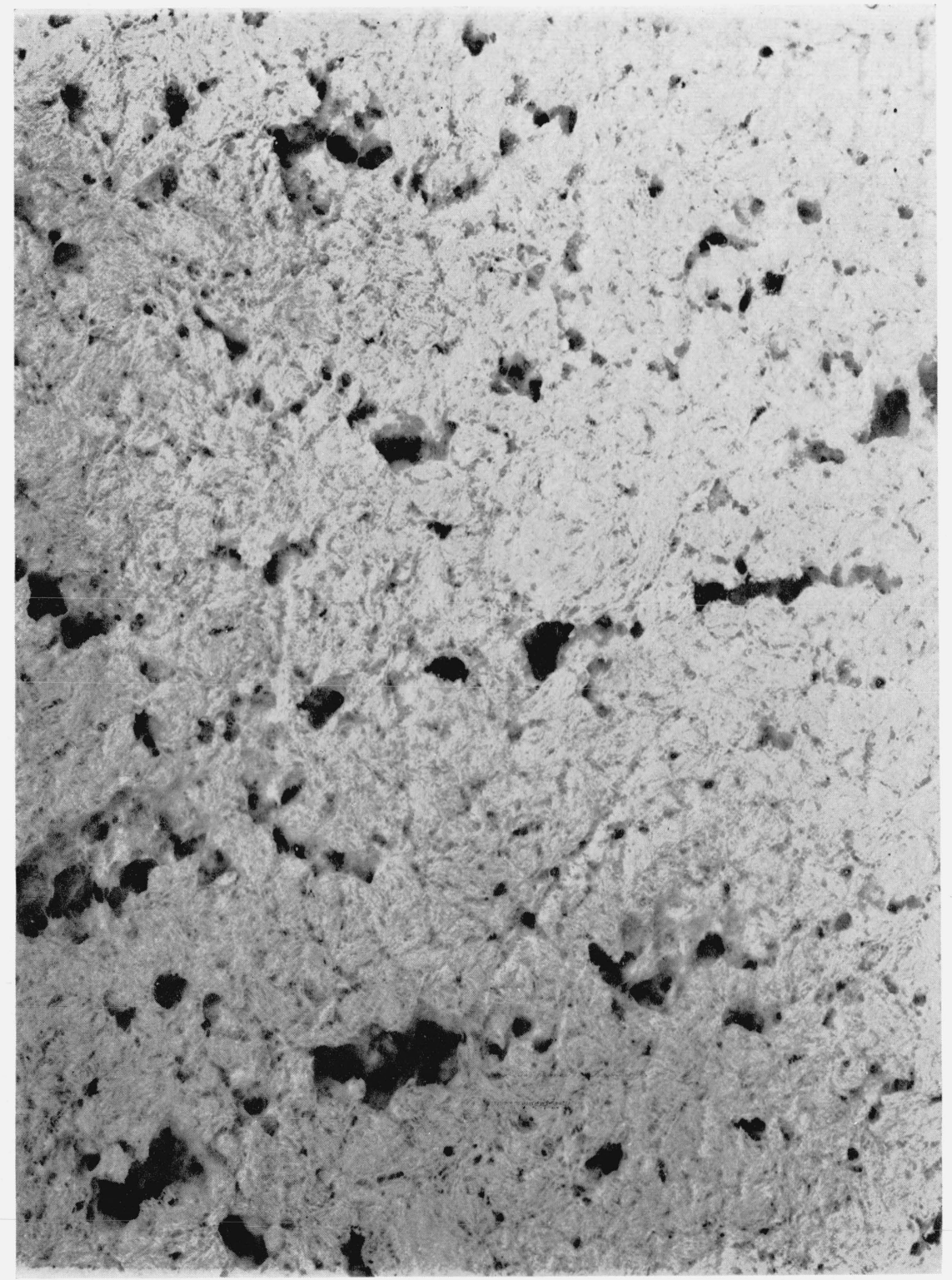

FIGURE 5.-Surface appearance of sample 3 when unpainted and magnified 12/3 times. 


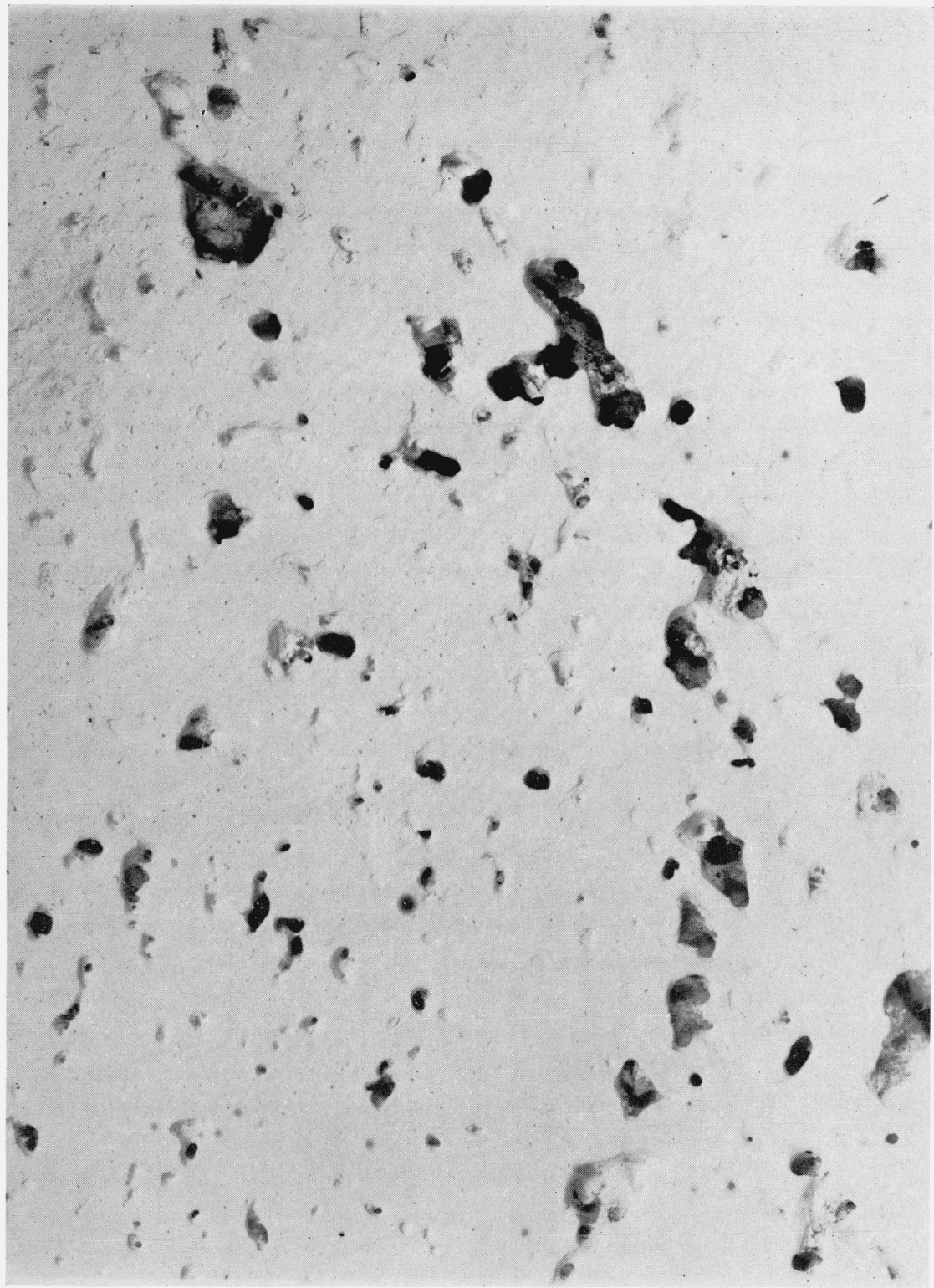

Figure 6.-Surface appearance of sample $3 a$ when brush painted five coats and magnified $1 \frac{2}{3}$ times. 


\begin{tabular}{|c|c|c|c|c|c|c|c|c|c|c|c|c|c|c|c|c|c|c|c|c|c|c|c|c|c|c|c|c|c|c|c|c|c|c|c|c|c|c|c|}
\hline & \multicolumn{2}{|c|}{$\bullet$} & 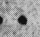 & \multirow{2}{*}{$\bullet$} & \multicolumn{2}{|c|}{$\bullet \bullet$} & & $\bullet$ & \multicolumn{2}{|c|}{$\bullet$} & $\bullet$ & & & & & & & & & • & & & $\bullet$ & & & & & • & • & $\bullet$ & & $\bullet$ & & & • & & & & \\
\hline & 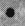 & - . & & & & & $\bullet$ & - & $\bullet$ & $\bullet$ & $\bullet$ & - & $\bullet$ & • & 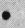 & • & & • & $\bullet$ & $\bullet$ & $\bullet$ & & • & & • & & $\bullet$ & $\bullet$ & • & • & 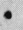 & $\bullet$ & & $\bullet$ & $\bullet$ & & & • & $\bullet$ \\
\hline 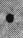 & 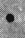 & - & - & - & - & - & - & $\bullet$ & $\bullet$ & - & - & $\bullet$ & • & - & • & • & & • & • & $\bullet$ & & & - & & $\bullet$ & & • & • & • & & & - & & • & • & & & - & - \\
\hline 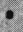 & - & - & - & - & $\bullet$ & - & $\bullet$ & $\bullet$ & $\bullet$ & • & $\bullet$ & $\bullet$ & $\bullet$ & $\bullet$ & $\bullet$ & $\bullet$ & & • & $\bullet$ & • & $\bullet$ & & - & & $\bullet$ & & • & $\bullet$ & - & & $\bullet$ & • & $\bullet$ & • & & & & • & - \\
\hline$\bullet$ & $\cdot$ & - & $\bullet$ & $\bullet$ & $\bullet$ & - & $\bullet$ & $\bullet$ & $\bullet$ & $\bullet$ & • & $\bullet$ & • & • & • & • & $\bullet$ & • & • & • & & & & & • & & •. & • & • & - & • & • & • & • & • & • & • & $\bullet$ & $\bullet$ \\
\hline - & • & • & - & $\bullet$ & • & - & $\bullet$ & $\bullet$ & - & • & • & $\bullet$ & • & - & • & • & & $\bullet$ & $\bullet$ & • & & & & & • & & $\bullet$ & $\bullet$ & • & • & $\bullet$ & • & • & • & & & & • & • \\
\hline - & $\bullet$ & 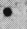 & - & $\bullet$ & $\bullet$ & - & - & $\bullet$ & • & • & • & $\bullet$ & $\bullet$ & • & • & • & 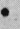 & • & • & - & & & & & • & & • & $\bullet$ & • & - & • & • & $\bullet$ & • & • & • & ? & $\bullet$ & - \\
\hline • & - & - & $\bullet$ & $\bullet$ & - & - & $\bullet$ & • & • & 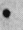 & • & $\bullet$ & $\bullet$ & $\bullet$ & $\bullet$ & • & & • & $\bullet$ & $\bullet$ & & & & & $\bullet$ & & • & • & • & $\bullet$ & $\bullet$ & • & • & • & • & & & $\bullet$ & $\bullet$ \\
\hline - & - & - & - & $\bullet$ & • & • & $\bullet$ & $\bullet$ & $\bullet$ & $\bullet$ & • & $\bullet$ & • & •. & $\bullet$ & • & $\bullet$ & • & $\bullet$ & • & & & 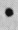 & & $\bullet$ & & • & • & $\bullet$ & • & $\bullet$ & $\bullet$ & - & $\bullet$ & $\bullet$ & & & $\bullet$ & - \\
\hline • & • & - & - & - & $\bullet$ & - & $\bullet$ & $\bullet$ & 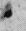 & $\bullet$ & • & $\bullet$ & 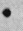 & • & • & • & & •. & $\bullet$ & • & & & & & $\bullet$ & & • & $\bullet$ & • & $\bullet$ & • & $\bullet$ & • & • & • & & & $\bullet$ & • \\
\hline$\bullet$ & - & - & - & $\bullet$ & - & $\bullet$ & $\bullet$ & $\bullet$ & • & • & •. & • & • & • & - & • & $\bullet$ & $\bullet$ & • & • & & & 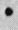 & & • & 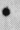 & • & • & • & $\bullet$ & • & • & $\bullet$ & & • & & • & $\bullet$ & • \\
\hline • & - & • & $\bullet$ & $\bullet$ & $\bullet$ & • & $\bullet$ & •. & $\bullet$ & $\bullet$ & • & $\bullet$ & $\bullet$ & • & $\bullet$ & • & & • & $\bullet$ & $\bullet$ & & & & & • & 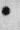 & • & • & • & $\bullet$ & $\bullet$ & • & $\bullet$ & $\bullet$ & • & & • & $\bullet$ & $\bullet$ \\
\hline • & • & - & - & $\cdot$ & $\bullet$ & $\bullet$ & $\bullet$ & • & - & ! & • & $\bullet$ & • & • & • & • & , & $\bullet$ & • & $\bullet$ & & & 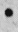 & & 4 & 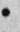 & • & • & • & - & • & • & • & $\bullet$ & - & & & $\bullet$ & • \\
\hline • & $\bullet$ & • & - & 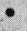 & - & $\bullet$ & - & $\bullet$ & - & • & - & • & • & • & $\bullet$ & • & & $\bullet$ & • & $\bullet$ & & & 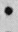 & & • & & • & • & • & - & $\bullet$ & • & $\bullet$ & $\bullet$ & - & & & $\bullet$ & $\bullet$ \\
\hline - & - & - & $\bullet$ & $\bullet$ & - & - & $\bullet$ & - & - & • & • & - & - & • & • & • & & • & • & - & & & 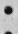 & & 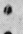 & 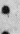 & - & • & • & - & - & - & - & $\bullet$ & - & & & - & • \\
\hline • & $\bullet$ & - & • & - & $\bullet$ & - & $\bullet$ & $\bullet$ & • & $\bullet$ & • & • & - & • & $\bullet$ & • & & • & • & - & & & 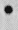 & & • & & • & • & • & • & • & • & • & • & - & & & $\bullet$ & $\bullet$ \\
\hline • & - & • & - & $\bullet$ & - & - & - & - & - & - & • & $\bullet$ & $\cdot$ & • & • & • & & • & 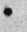 & $\bullet$ & & & 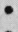 & & $\bullet$ & . & - & - & • & • & • & - & & $\bullet$ & - & & & $\bullet$ & $\bullet$ \\
\hline$\bullet$ & - & - & - & - & - & - & - & $\bullet$ & - & • & - & - & 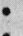 & • & - & • & $\bullet$ & • & • & • & & & 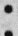 & & • & & • & • & • & • & - & • & • & • & • & & & $\bullet$ & \\
\hline • & : & • & ? & 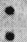 & : & - & $:$ & $\because$ & : & $\bullet$ & - & $\bullet$ & $\bullet$ & $\bullet$ & $\bullet$ & • & $\bullet$ & . & - & $\bullet$ & & $\Rightarrow$ & 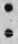 & 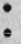 & • & 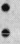 & - & : & • & $\bullet$ & : & & • & $\stackrel{\bullet}{\bullet}$ & & & & 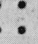 & $\dot{0}$ \\
\hline$\bullet$ & * & - & $\bullet$ & - & - & - & - & $\bullet$ & • & - & $\bullet$ & $\bullet$ & • & - & - & $\bullet$ & 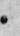 & - & - & $\bullet$ & & 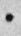 & 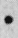 & $\bullet$ & - & 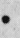 & • & • & - & • & • & - & - & • & • & & & $\bullet$ & 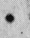 \\
\hline 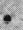 & & • & & & $\bullet$ & & $\bullet$ & & & & & & & & & & & & & & & & & & & & & & & & & & & & & & & • & \\
\hline •. & $\bullet$ & & - & $\bullet$ & $\bullet$ & - & $\bullet$ & $\bullet$ & $\bullet$ & $\bullet$ & $\bullet$ & $\bullet$ & $\bullet$ & $\bullet$ & • & 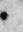 & $\bullet$ & $\bullet$ & 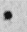 & 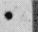 & & & & & & & & $\bullet$ & $\bullet$ & & 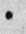 & • & - & 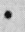 & - & • & & $\bullet$ & $\bullet$ \\
\hline • & - & - & • & - & - & - & $\bullet$ & $\bullet$ & $\bullet$ & $\bullet$ & • & $\bullet$ & • & $\bullet$ & • & 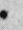 & & 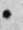 & 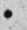 & 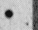 & & & & & • & & & • & - & & • & • & & $\bullet$ & & & & • & $\bullet$ \\
\hline • & - & • & • & $\bullet$ & - & - & $\bullet$ & - & - & $\bullet$ & • & • & (8) & • & $\bullet$ & 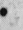 & & $\bullet$ & 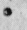 & 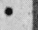 & & & & & & & & • & • & & $\bullet$ & $\bullet$ & & $\bullet$ & $\bullet$ & & & $\bullet$ & $\bullet$ \\
\hline - & - & 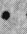 & $\bullet$ & - & - & - & $\bullet$ & - & - & $\bullet$ & - & • & • & - & - & - & & 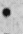 & $\bullet$ & $\bullet$ & & & & & $\bullet$ & & & • & • & & $\bullet$ & - & & $\bullet$ & & & & • & $\bullet$ \\
\hline 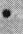 & - & - & $\bullet$ & $\bullet$ & - & - & $\bullet$ & $\bullet$ & - & •. & • & $\bullet$ & • & - & • & 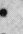 & $\bullet$ & • & - & 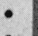 & & & & & & & • & • & - & & $\bullet$ & $\bullet$ & & - & $\bullet$ & & & $\bullet$ & $\bullet$ \\
\hline$\cdot$ & - & - & - & - & - & - & $\bullet$ & - & - & 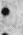 & 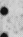 & $\bullet$ & $\bullet$ & - & 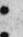 & $\bullet$ & & • & 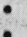 & 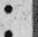 & & & & & & & • & • & $\bullet$ & & & & & & $\stackrel{\bullet}{\bullet}$ & & & • & $\bullet$ \\
\hline • & - & $\bullet$ & • & ?. & 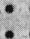 & - & 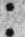 & $\bullet$ & $\bullet$ & • & $\bullet$ & 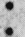 & & $\bullet$ & $\bullet$ & & & & 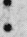 & & & & & & & & & $\bullet$ & 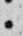 & & 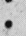 & & & & & & & - & \\
\hline 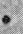 & - & 0 & $\bullet$ & $\bullet$ & • & - & $\bullet$ & 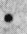 & $\bullet$ & • & • & - & & • & $\bullet$ & 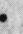 & 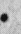 & - & 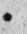 & 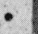 & & & & & & & & $\bullet$ & 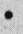 & & - & $\bullet$ & & - & $\cdot$ & & & $\bullet$ & 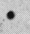 \\
\hline c. & - & $\bullet$ & - & $\bullet$ & • & - & $\bullet$ & 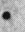 & 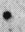 & 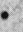 & 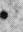 & 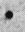 & & 3 & tes & 3 & & 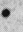 & & t & & & & & - & & • & - & • & & $\bullet$ & & & $\bullet$ & & & & • & c \\
\hline 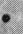 & • & - & • & a & - & - & $\bullet$ & 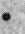 & $\bullet$ & $\bullet$ & - & $\bullet$ & 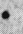 & - & • & 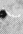 & & 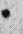 & 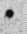 & - & & & & & - & & & - & - & & $\bullet$ & - & & $\bullet$ & - & - & & $\bullet$ & - \\
\hline$\bullet$ & - & - & • & $\bullet$ & • & - & • & • & • & - & - & - & 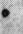 & - & 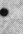 & 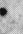 & & (1) & . & - & & & & & • & & 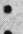 & - & - & & $\bullet$ & $\bullet$ & & 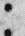 & $\bullet$ & & & $\bullet$ & $\bullet$ \\
\hline • & $\bullet$ & - & • & $\bullet$ & • & • & - & $\bullet$ & $\bullet$ & - & - & - & 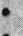 & 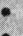 & & & • & & & • & & & & & • & & $\bullet$ & • & $\bullet$ & & - & - & & - & - & & & - & $\bullet$ \\
\hline - & - & • & $\bullet$ & $\bullet$ & $\bullet$ & $\bullet$ & $\bullet$ & 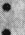 & $\bullet$ & - & 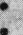 & $\bullet$ & 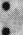 & 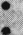 & $\bullet$ & 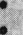 & & 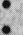 & 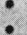 & 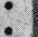 & & & & & 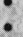 & & & $\bullet$ & 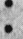 & - & & & & & & & & - & - \\
\hline$\bullet$ & $\bullet$ & • & $\bullet$ & $\bullet$ & - & $\bullet$ & $\bullet$ & 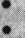 & & 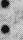 & 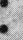 & 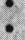 & 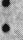 & 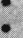 & 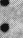 & & 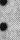 & & & & & & & & 7 & & & 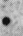 & 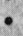 & & & & & & & & & $\bullet$ & \\
\hline 6 & $\bullet$ & 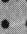 & - & $\bullet$ & $\bullet$ & $\bullet$ & $\bullet$ & 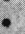 & 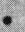 & & ? & 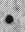 & 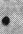 & 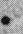 & & - & 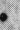 & 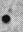 & 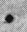 & 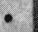 & & & & & 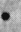 & & & 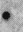 & 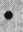 & & & & & 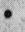 & & & & 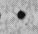 & - \\
\hline$\bullet$ & . & . & - & 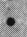 & - & - & 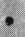 & 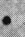 & - & . & 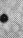 & * & 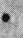 & 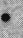 & 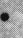 & 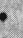 & & 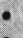 & 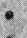 & 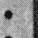 & & & & & 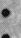 & & 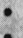 & - & • & & . & & . & $\bullet$ & . & & & . & - \\
\hline • & - & $\bullet$ & 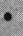 & 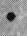 & $\bullet$ & - & 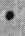 & 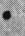 & 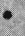 & 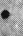 & 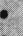 & 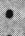 & 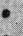 & 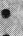 & 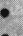 & 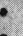 & 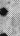 & - & , & 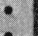 & & & 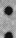 & & - & 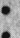 & & & • & & & • & - & 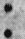 & - & - & & $\bullet$ & $\bullet$ \\
\hline$\bullet$ & $\bullet$ & $\bullet$ & •. & - & $\bullet$ & $\bullet$ & - & & $\bullet$ & & & & & & & & & ? & & $\bullet$ & & 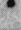 & & & & & & & & & & & 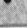 & & & & & 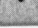 & \\
\hline
\end{tabular}

Figure 7.-General appearance of sample 4. 


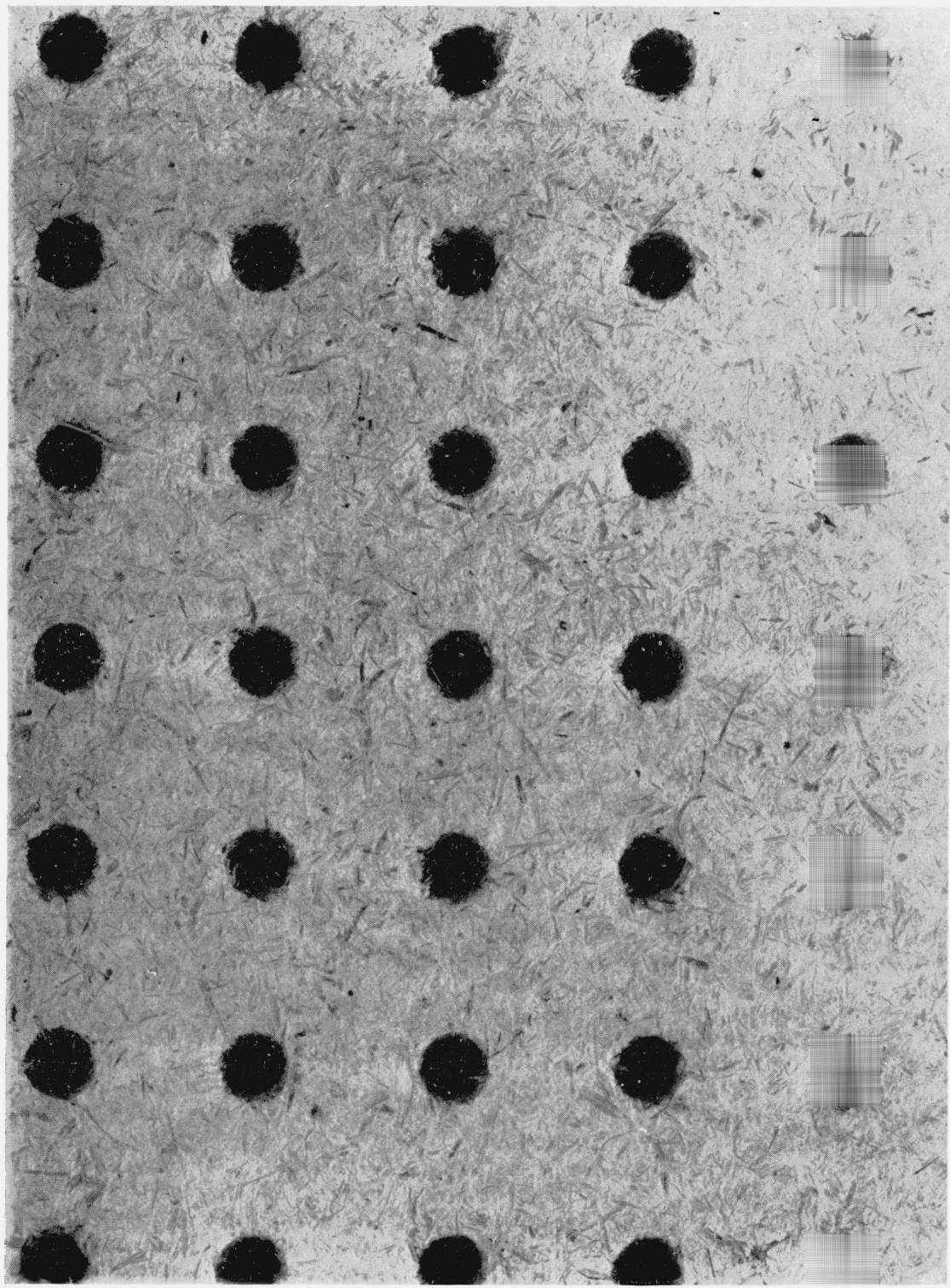

Figure 8.- Surface appearance of sample 4 with $R$. I. finish when magnified 12/3 times. 


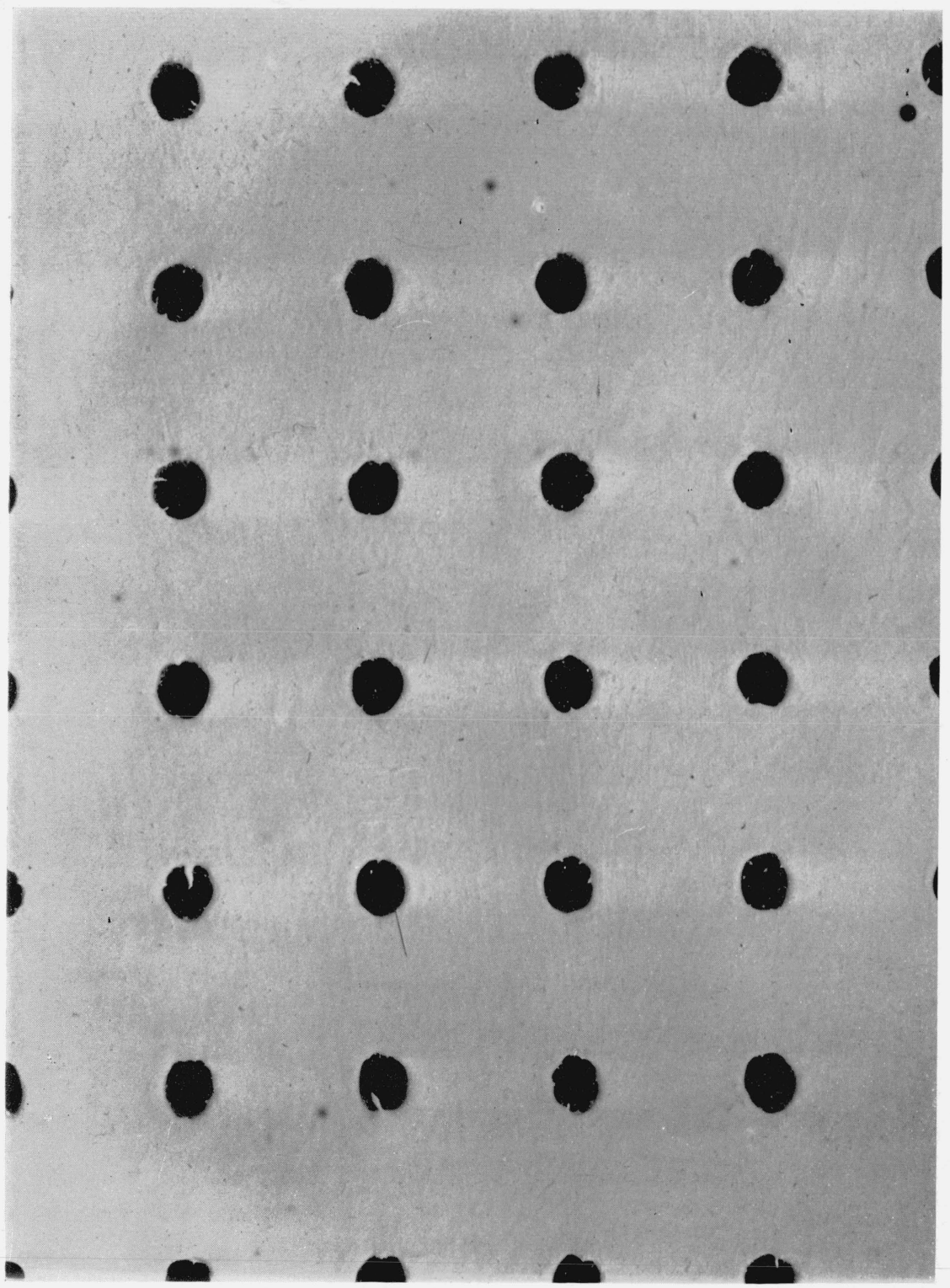

Figure 9.-Surface appearance of sample $4 a$ when brush painted four coats and magnified $1^{2} \frac{2}{3}$ times. 


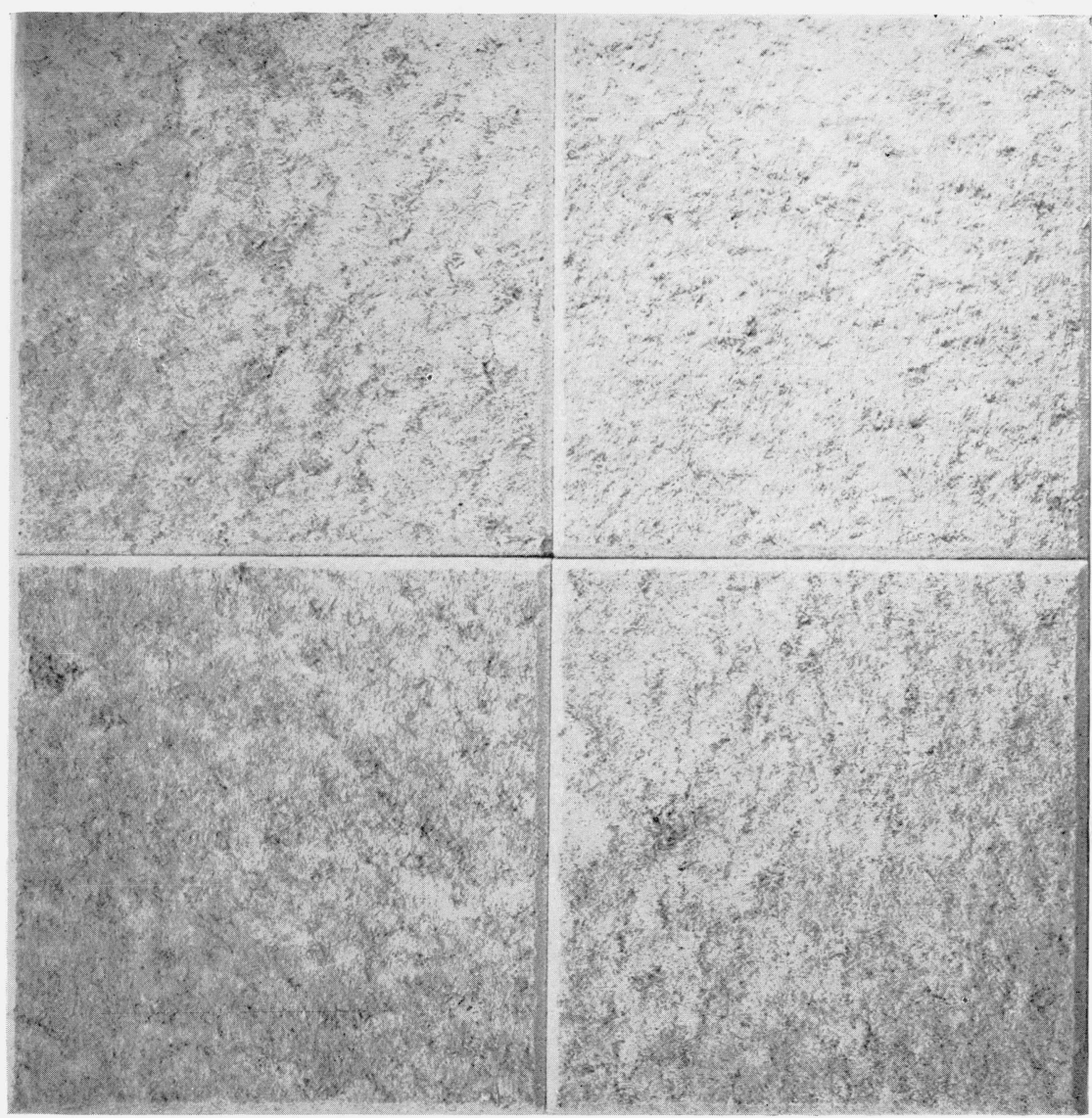

Figure 10.-General appearance of sample 5. 


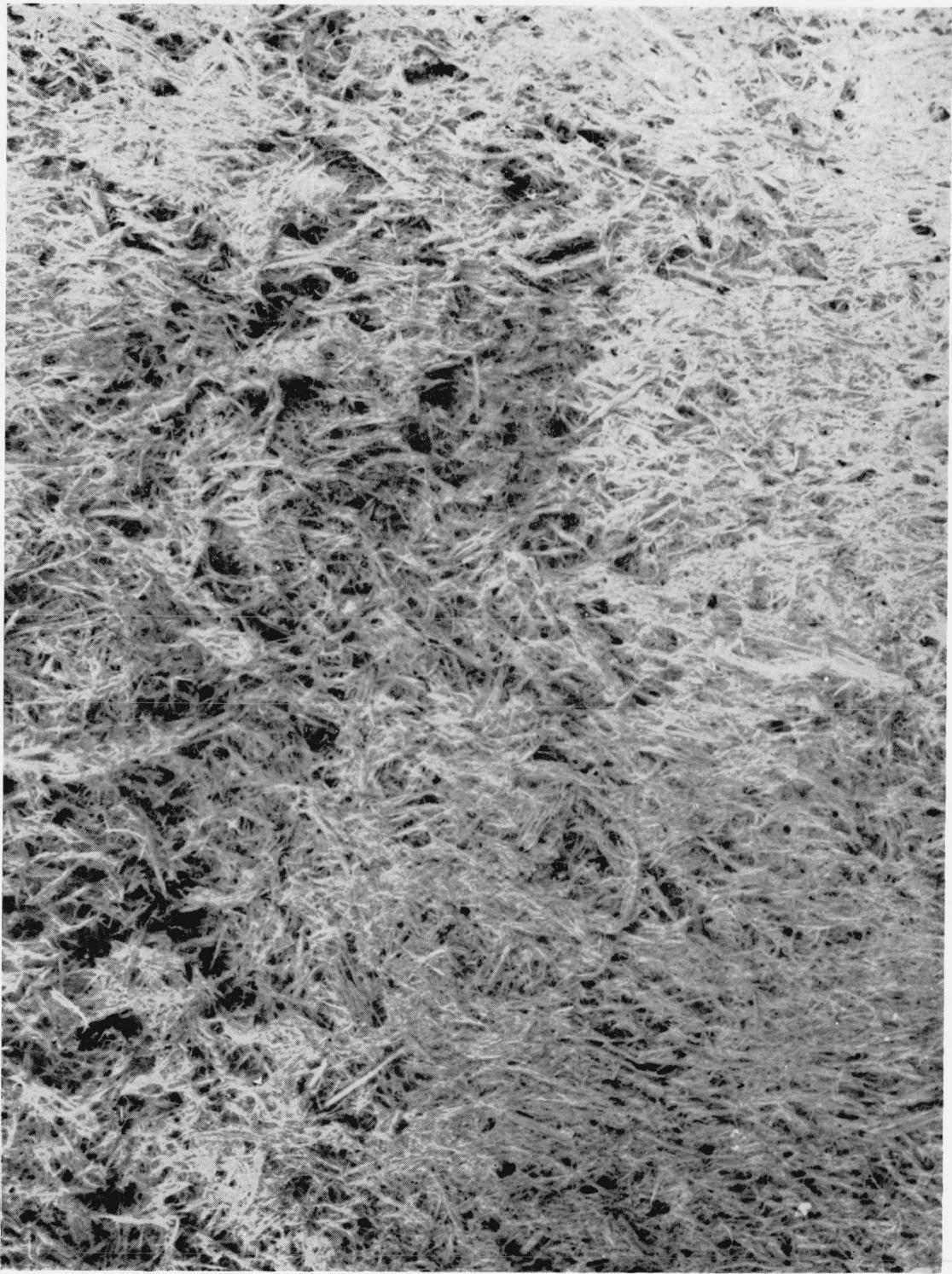

FIGURE 11.-Surface appearance of sample 5 when unpainted and magnified three times. 


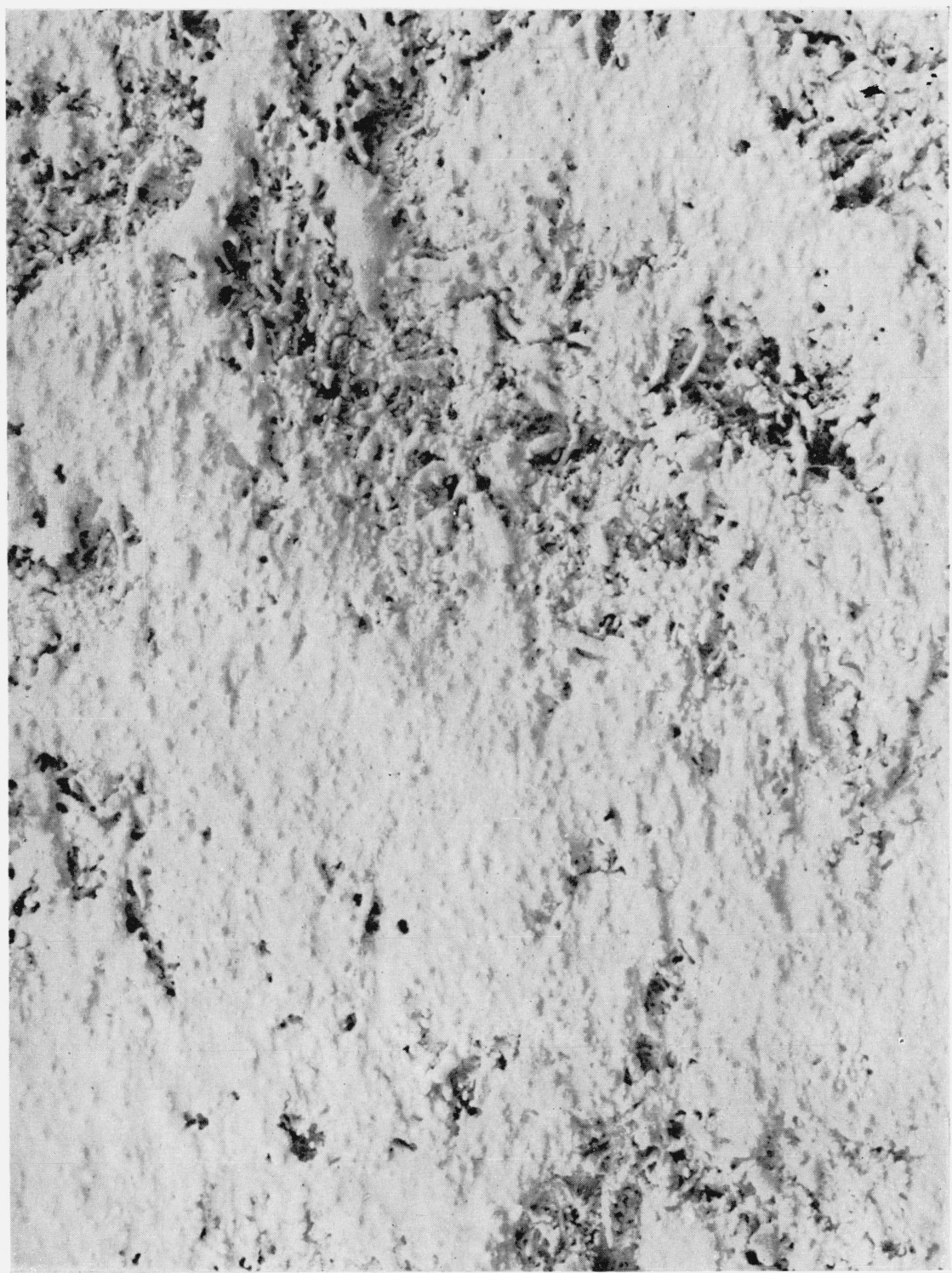

FIGURE 12.-Surface appearance of sample 5a when spray painted seven coats and magnified three times. 


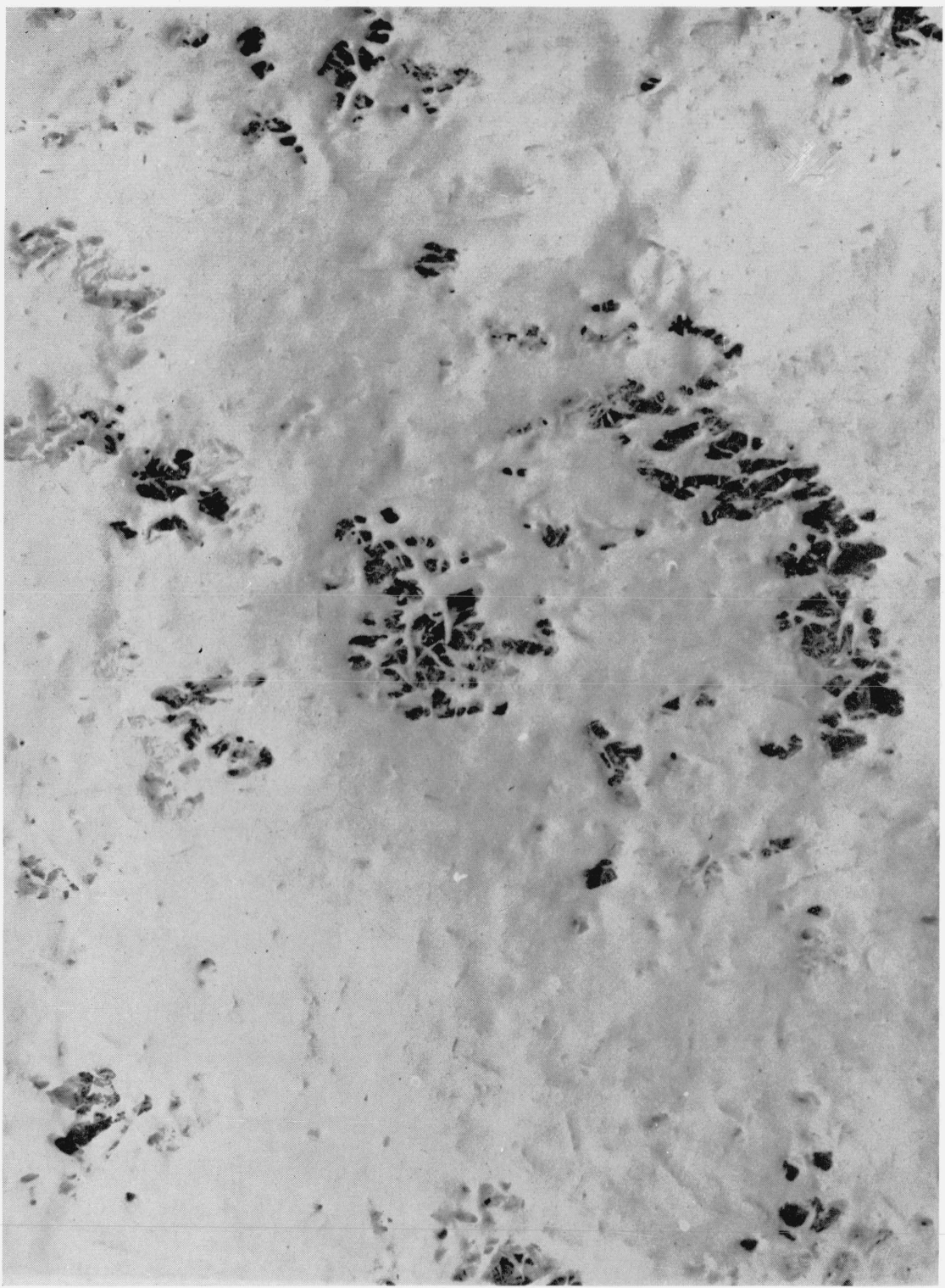

FIGURE 13.- Surface appearance of sample $5 b$ when brush painted five coats and magnified three times. 


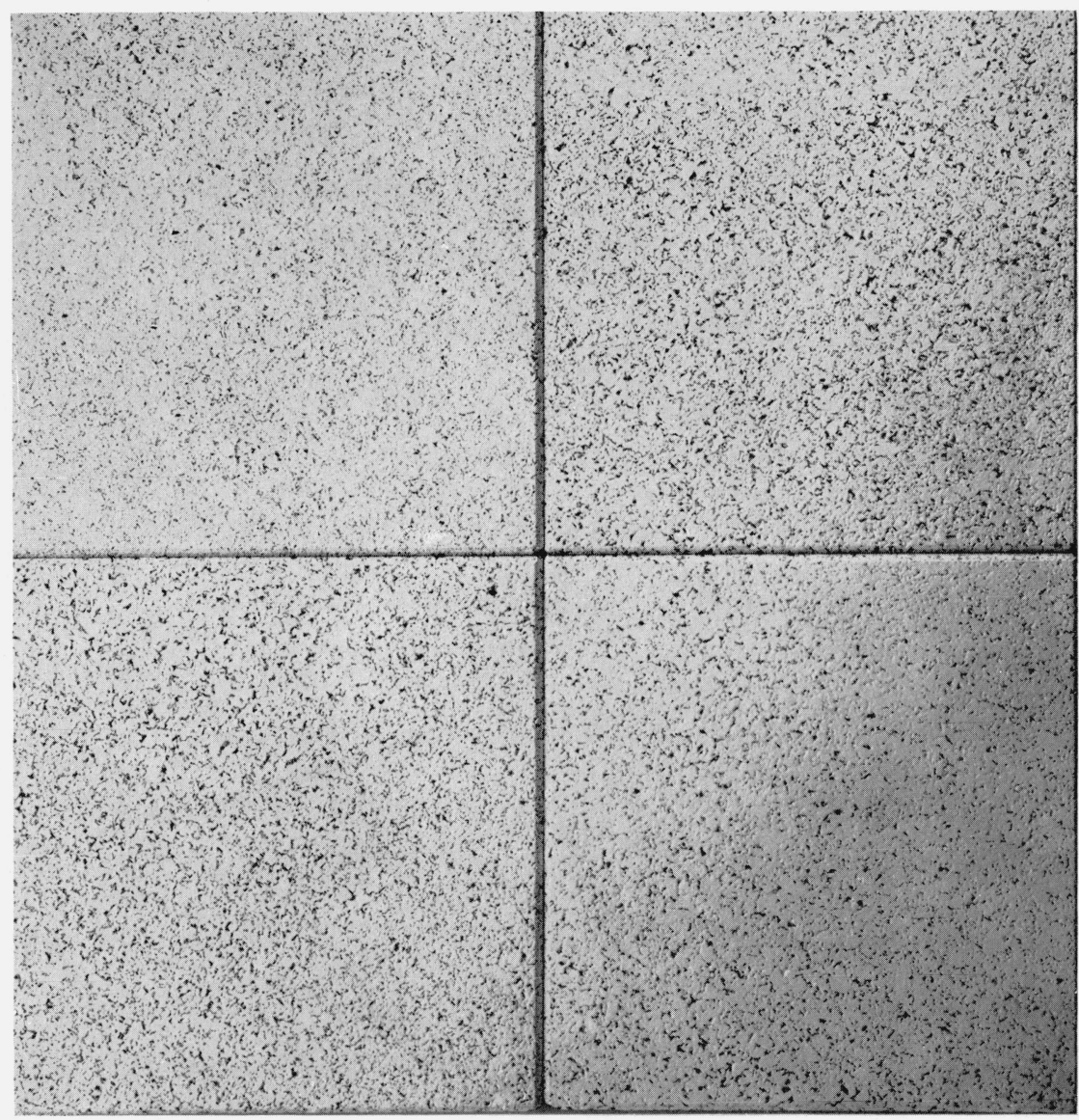

Figure 14.-General appearance of sample 6. 


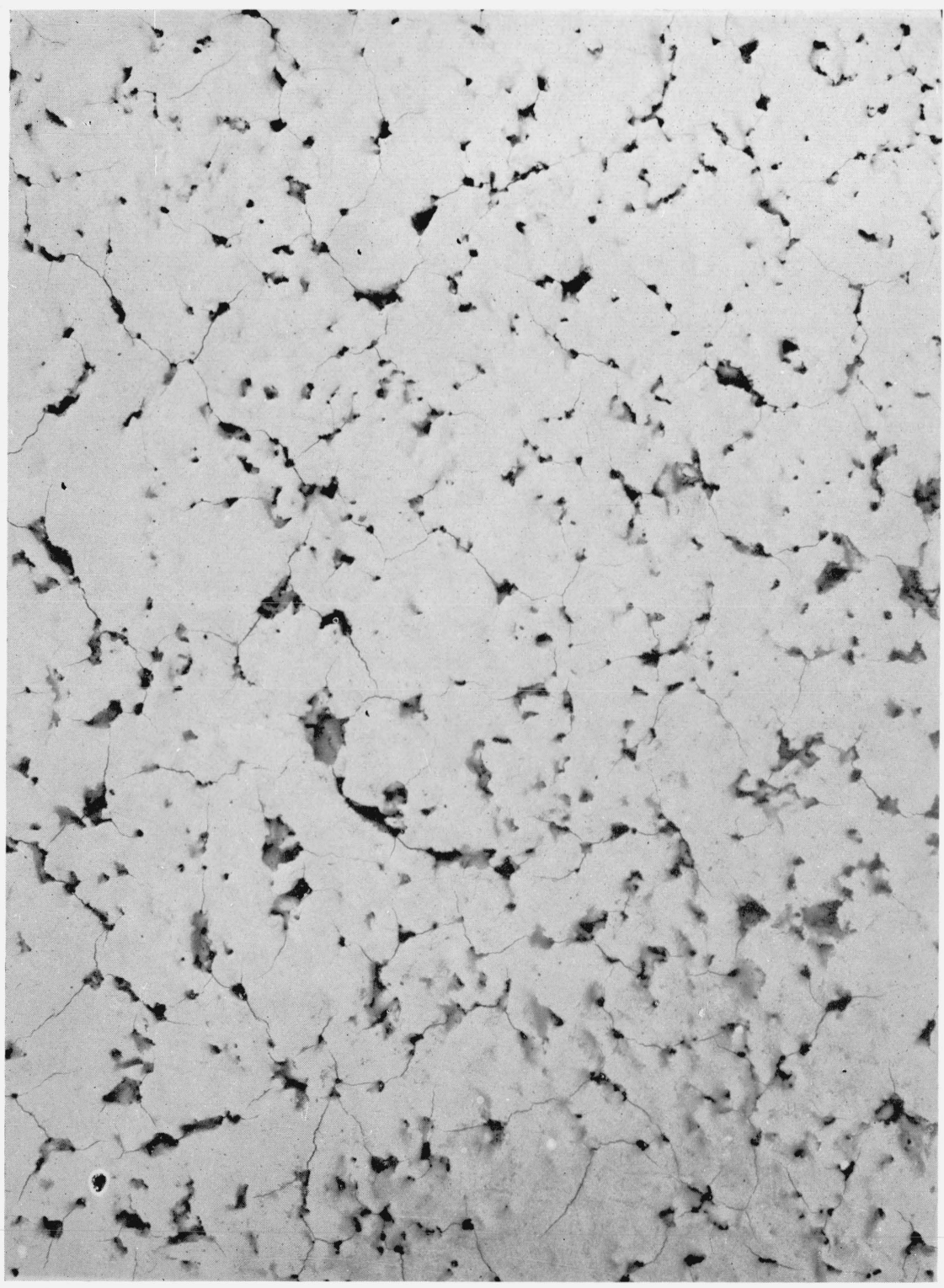

Figure 15.-Surface appearance of sample 6 as painted by manufacturer when magnified $1^{2} \frac{3}{3}$ times. 


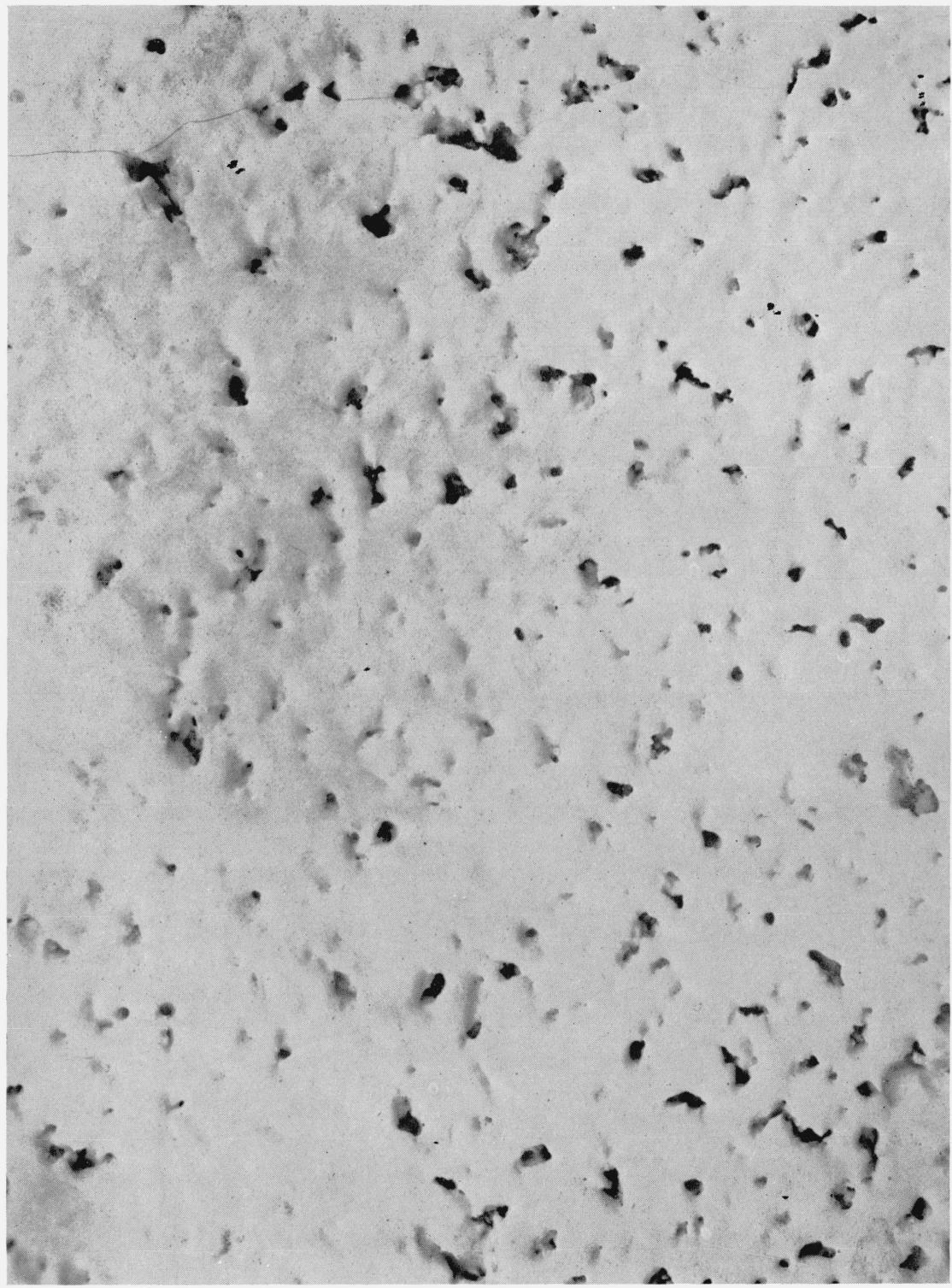

FIGURE 16.-Surface appearance of sample 6 a when brush painted four additional coats and magnified $1 \frac{2}{3}$ times. 


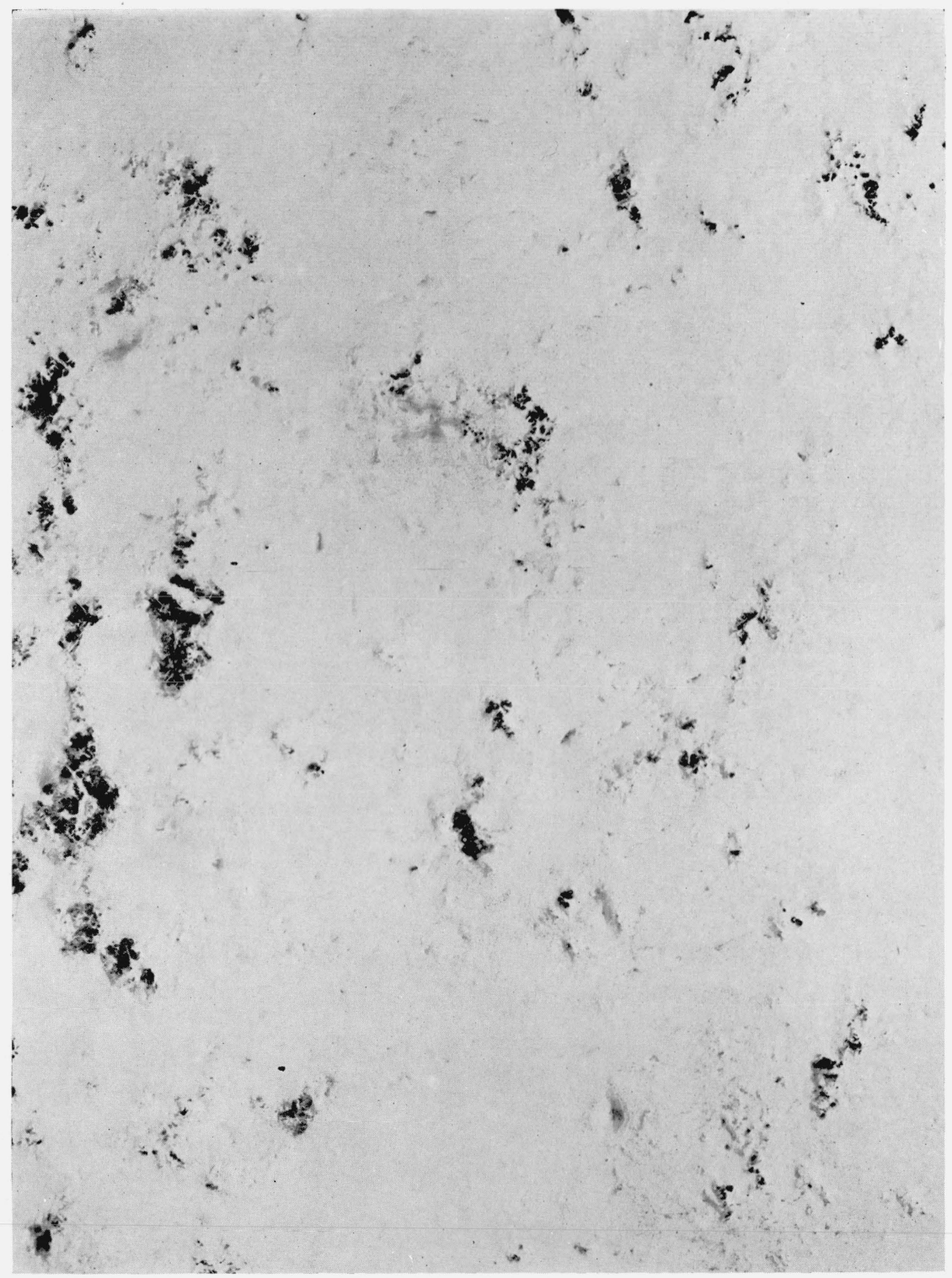

FigURE 17.- Surface appearance of sample 7 a when brush painted four additional coats and magnified two-thirds times. 


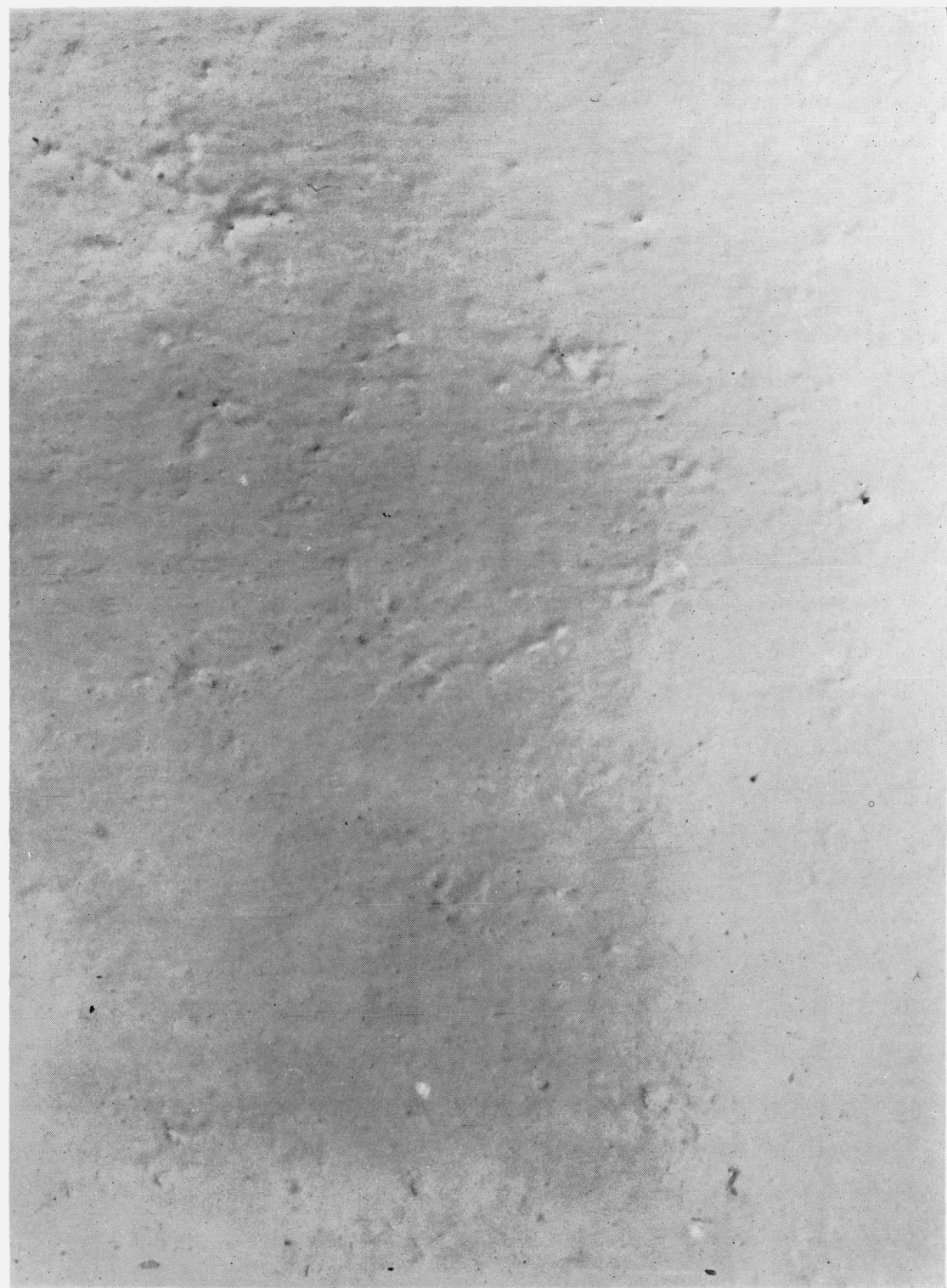

FIGURE 18.- Surface appearance of sample 8 a when brush painted five additional coats and magnified two-thirds times. 


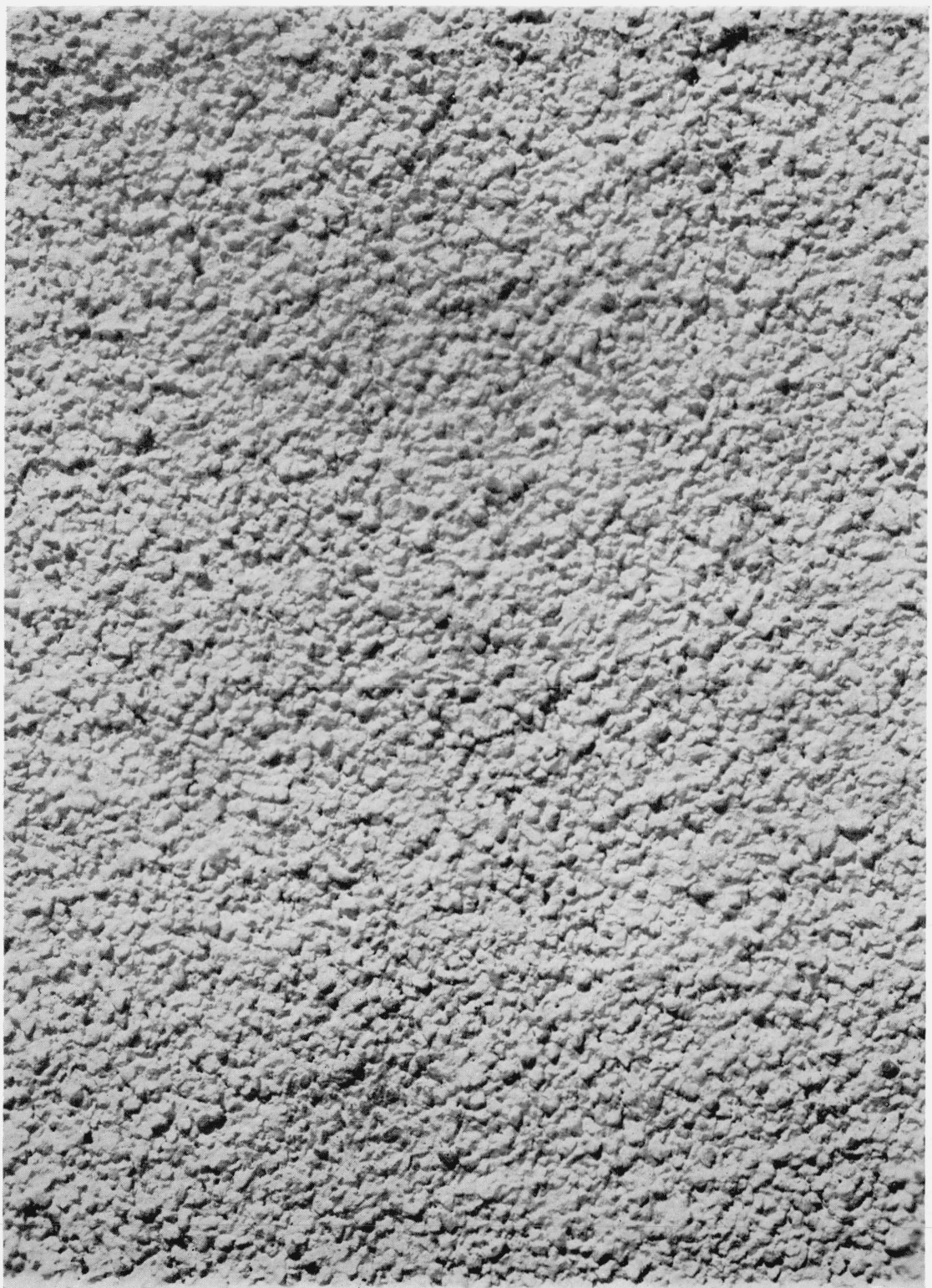

Figure 19.-Surface appearance of sample 9 when magnified $1^{2 / 3}$ times. 


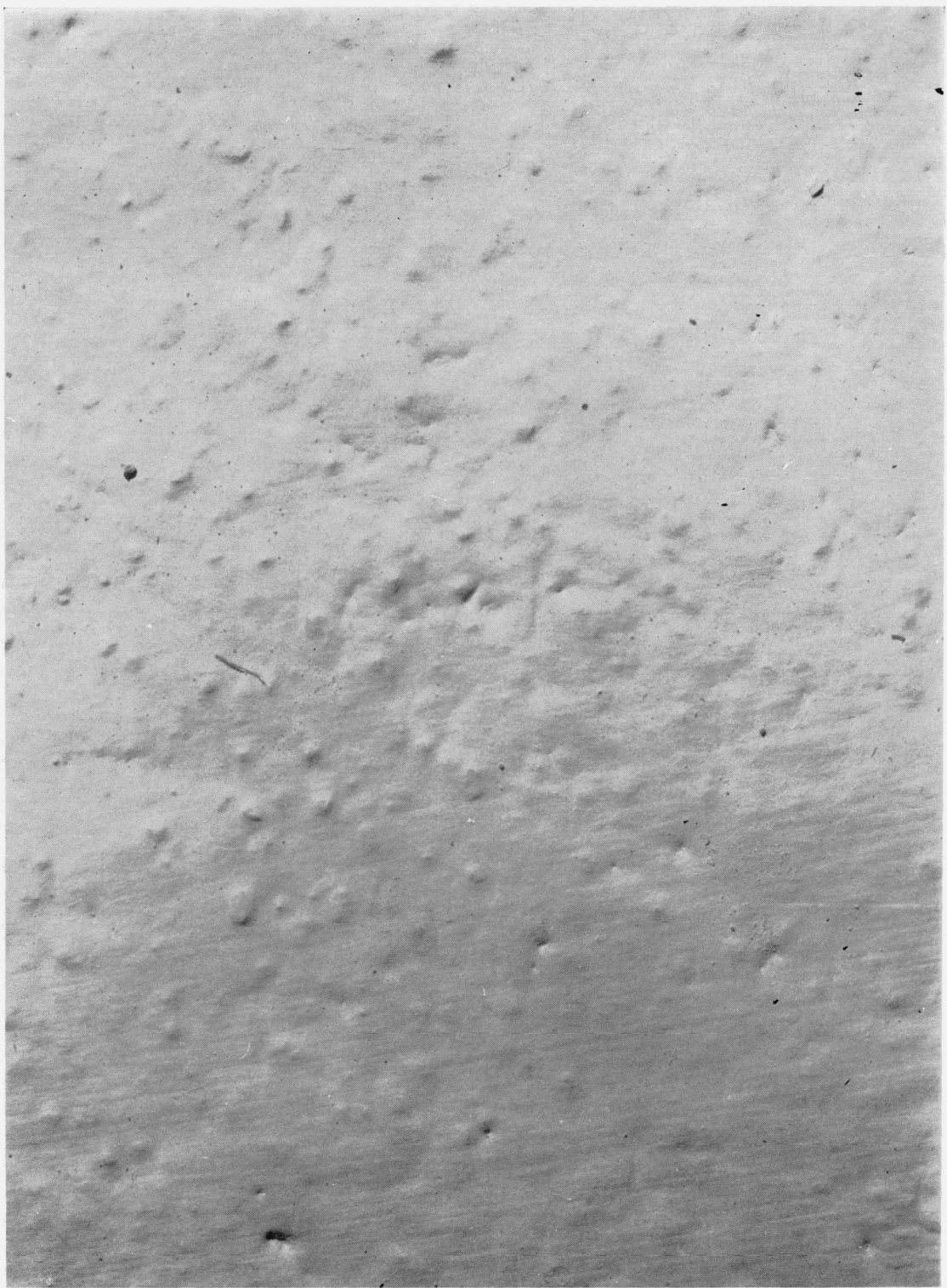

FiguRE 20. - Surface appearance of sample $9 a$ when brush painted five coats and magnified $1 \frac{2}{3}$ times. 
opening. When the pore openings are larger, more coats of paint are required before this deposit of pigment will close the openings.

The manner in which the paint is applied is also important, that is, whether the paint is applied with a brush or with a spray gun. It is possible to obtain a more uniform coating of the surface particles or projections when the paint is applied with a spray gun than when applied with a brush; likewise, more coats of paint are required before the pigment builds across the pore openings.

TABLE 1.-Sound absorption coefficients of test panels

\begin{tabular}{|c|c|c|c|c|c|c|c|c|}
\hline \multirow{2}{*}{ Sample } & \multirow{2}{*}{ Surface finish } & \multicolumn{6}{|c|}{$\begin{array}{l}\text { Sound absorption coefficients at } \\
\text { frequencies (cycles per second)- }\end{array}$} & \multirow{2}{*}{$\begin{array}{l}\text { Noise } \\
\text { coeffi- } \\
\text { cients }\end{array}$} \\
\hline & & 128 & 256 & 512 & 1,024 & 2,048 & 4,096 & \\
\hline 1 & $\begin{array}{l}\text { Unpainted } \\
\text { Brush painted } 5 \text { coats. }\end{array}$ & $\begin{array}{r}0.09 \\
.14\end{array}$ & $\begin{array}{r}0.24 \\
.49\end{array}$ & $\begin{array}{r}0.62 \\
.83\end{array}$ & $\begin{array}{r}0.89 \\
.61\end{array}$ & $\begin{array}{r}0.73 \\
.30\end{array}$ & $\begin{array}{r}0.73 \\
.22\end{array}$ & $\begin{array}{r}0.60 \\
.55\end{array}$ \\
\hline $2 a_{-}^{2 .}$ & $\begin{array}{l}\text { Painted by manufacturer } \\
\text { Brush painted } 5 \text { additional coats.- }\end{array}$ & $\begin{array}{l}.07 \\
.09\end{array}$ & .22 & .54 & $\begin{array}{l}.87 \\
.92\end{array}$ & $\begin{array}{l}.78 \\
.62\end{array}$ & $\begin{array}{l}.77 \\
.62\end{array}$ & $\begin{array}{r}.60 \\
.65\end{array}$ \\
\hline $\begin{array}{l}3 .- \\
s a_{-}\end{array}$ & $\begin{array}{l}\text { Unpainted } \\
\text { Brush painted } 5 \text { coats. }\end{array}$ & .11 & $\begin{array}{l}.29 \\
.42\end{array}$ & .78 & .84 & $\begin{array}{l}.75 \\
.61\end{array}$ & $\begin{array}{l}.63 \\
.49\end{array}$ & $\begin{array}{l}.65 \\
.65\end{array}$ \\
\hline & $\begin{array}{l}\text { R. I. finish } \\
\text { Brush painted } 4 \text { coats. }\end{array}$ & $\begin{array}{l}.10 \\
.08\end{array}$ & $\begin{array}{l}.30 \\
.33\end{array}$ & .78 & .85 & $\begin{array}{l}.59 \\
.53\end{array}$ & $\begin{array}{l}.42 \\
.37\end{array}$ & $\begin{array}{l}.65 \\
.65\end{array}$ \\
\hline $\begin{array}{l}5-. \\
5 a_{-} \\
5 b_{-}\end{array}$ & $\begin{array}{l}\text { Unpainted } \\
\text { Spray painted } 7 \text { coats. } \\
\text { Brush painted } 5 \text { coats }\end{array}$ & $\begin{array}{l}.14 \\
.18 \\
.25\end{array}$ & $\begin{array}{l}.51 \\
.50 \\
.41\end{array}$ & $\begin{array}{l}.78 \\
.79 \\
.61\end{array}$ & $\begin{array}{l}.78 \\
.74 \\
.47\end{array}$ & $\begin{array}{l}.78 \\
.71 \\
.34\end{array}$ & $\begin{array}{l}.82 \\
.67 \\
.36\end{array}$ & $\begin{array}{l}.70 \\
.70 \\
.45\end{array}$ \\
\hline $6 a_{-}$ & $\begin{array}{l}\text { Painted by manufacturer } \\
\text { Brush painted } 4 \text { additional coats... }\end{array}$ & .07 & .25 & $\begin{array}{l}.70 \\
.66\end{array}$ & $\begin{array}{l}.63 \\
.45\end{array}$ & .53 & $\begin{array}{l}.63 \\
.39\end{array}$ & $\begin{array}{l}.55 \\
.45\end{array}$ \\
\hline$\gamma_{a_{-}-.}$ & $\begin{array}{l}\text { Unpainted } \\
\text { Brush painted } 6 \text { coats. }\end{array}$ & .54 & .87 & .78 & .73 & .78 & .75 & .80 \\
\hline $8 a_{-\ldots}$ & $\begin{array}{l}\text { Painted by manufacturer } \\
\text { Brush painted } 4 \text { coats. }\end{array}$ & $\begin{array}{l}.57 \\
.58\end{array}$ & .71 & .80 & $\begin{array}{l}.56 \\
.28\end{array}$ & .51 & .52 & $\begin{array}{l}.65 \\
.45\end{array}$ \\
\hline $9 a_{-}$ & $\begin{array}{l}\text { Painted by manufacturer } \\
\text { Brush painted } 5 \text { additional coats..- }\end{array}$ & .13 & .40 & .68 & .63 & $\begin{array}{r}.60 \\
.18\end{array}$ & $\begin{array}{l}.68 \\
.23\end{array}$ & $\begin{array}{r}.60 \\
.25\end{array}$ \\
\hline
\end{tabular}

TABLE 2.-Amount of paint applied per coat to test panels

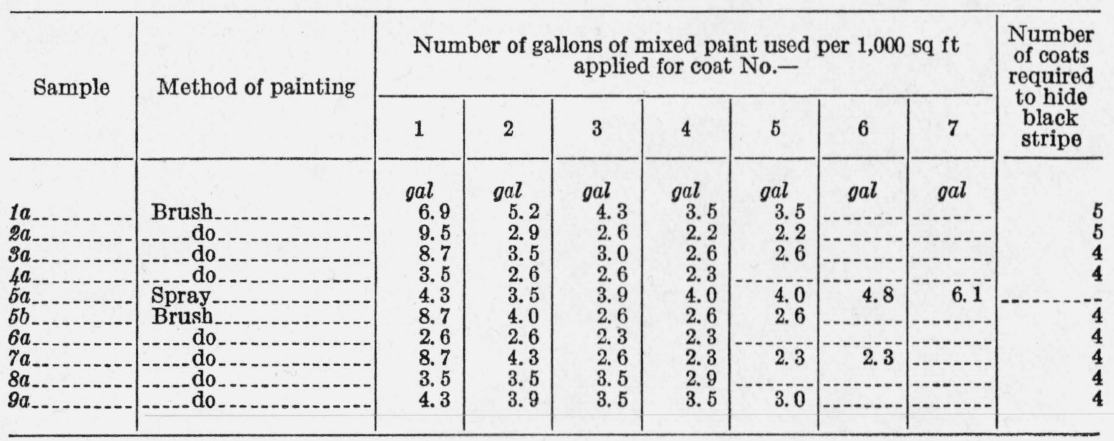

Washington, March 12, 1940 